\title{
Differential Stability of Two-Stage Stochastic Programs*
}

\author{
Darinka Dentcheva and Werner Römisch \\ Humboldt-Universität Berlin \\ Institut für Mathematik \\ Unter den Linden 6 \\ 10099 Berlin, Germany
}

\begin{abstract}
Two-stage stochastic programs with random right-hand side are considered. Optimal values and solution sets are regarded as mappings of the expected recourse functions and their perturbations, respectively. Conditions are identified implying that these mappings are directionally differentiable and semidifferentiable on appropriate functional spaces. Explicit formulas for the derivatives are derived. Special attention is paid to the role of a Lipschitz condition for solution sets as well as of a quadratic growth condition of the objective function.
\end{abstract}

Keywords: Two-stage stochastic programs, sensitivity analysis, directional derivatives, semidifferentiability, solution sets.

1991 MSC: 90 C 15, 90 C 31

\section{Introduction}

Two-stage stochastic programming is concerned with problems that require a hereand-now decision on the basis of given probabilistic information on the random data without making further observations. The costs to be minimized consist of the direct costs of the here-and-now (or first stage) decision as well as the costs generated by the need of taking a recourse (or second stage) decision in response to the random environment. Recourse costs are often formulated by means of expected values with respect to the probability distribution of the involved random data. In this way, two-stage models and their solutions depend on the underlying probability distribution. Since this distribution is often incompletely known in applied models, or it has to be approximated for computational purposes, the stability behaviour of stochastic programming models when changing the probability measure is important. This problem is studied in a number of papers. We only mention here the surveys [13], [37] and the papers [1],

\footnotetext{
${ }^{*}$ This research is supported by the Deutsche Forschungsgemeinschaft
} 
[12], [17], [24], [25], [31] and [32]. The paper [1] contains general results on continuity properties of optimal values and solutions when perturbing the probability measures with respect to the topology of weak convergence. Quantitative continuity results of solution sets to two-stage stochastic programs with respect to suitable distances of probability measures are derived in [24] and [25]. Asymptotic properties of statistical estimators of values and solutions to stochastic programs are derived in [17], [31], [32]. They are based on directional differentiability properties of the underlying optimization problems with respect to the parameter that carries the randomness $([17],[32])$ or the probability measure ([31]). These directional differentiability results for values (in [32]) and solutions (in [17], [31]) lead to asymptotic results via the so-called delta-method. For a description of the delta-method we refer to Chapter 6 in [26], [32], to [33] for an up-to-date presentation and to [15] for a set-valued variant. These papers illuminate the importance of the Hadamard directional differentiability (for single-valued functions) and of the semidifferentiability (for set-valued mappings) in the context of asymptotic statistics.

The present paper aims at contributing to this line of differential stability studies. The results in [17], [31] apply to fairly general stochastic optimization models, but impose conditions that are rather restrictive in our context. The present paper deals with special two-stage models and, using structural properties, avoids certain assumptions that complicate or even prevent the applicability of those general results to two-stage stochastic programs. Such assumptions are the (local) uniqueness of solutions and differentiability properties of perturbed problems, which are indispensable in [17], [31]. Before discussing this in more detail, let us introduce the class of two-stage stochastic programs, we want to consider:

$$
\min \left\{g(x)+Q_{\mu}(A x): x \in C\right\},
$$

where $g: \mathbb{R}^{m} \rightarrow \mathbb{R}$ is a convex function, $C \subseteq \mathbb{R}^{m}$ is a nonempty closed convex set, $A$ is a $(s, m)$-matrix and $Q_{\mu}$ is the expected recourse function with respect to the (Borel) probability measure $\mu$ on $\mathbb{R}^{s}$,

$$
\begin{aligned}
Q_{\mu}(y) & =\int_{\mathbb{R}^{s}} \tilde{Q}(\zeta-y) \mu(\mathrm{d} \zeta), \\
\tilde{Q}(t) & =\inf \{\langle q, u\rangle: W u=t, u \geq 0\} \quad\left(t \in \mathbb{R}^{s}\right) .
\end{aligned}
$$

Here $q \in \mathbb{R}^{\bar{m}}$ are the recourse costs, $W$ is an $(s, \bar{m})$-matrix and called the recourse matrix, and $\tilde{Q}(\zeta-A x)$ corresponds to the value of the optimal second stage decision for compensating a possible violation of the (random) constraint $A x=\zeta$. To have the problem (1.1) - (1.3) well-defined, we assume

$$
\begin{array}{ll}
\operatorname{pos} W=\left\{W u: u \in \mathbb{R}_{+}^{\bar{m}}\right\}=\mathbb{R}^{s} & \text { (complete recourse) } \\
M_{D}=\left\{t \in \mathbb{R}^{s}: W^{T} t \leq q\right\} \neq \emptyset & \text { (dual feasibility) } \\
\int_{\mathbb{R}^{s}}\|\zeta\| \mu(\mathrm{d} \zeta)<\infty & \text { (finite first moment). }
\end{array}
$$

The assumptions (A1) and (A2) imply that $\tilde{Q}$ is finite, convex and polyhedral on $\mathbb{R}^{s}$. Due to (A3) also $Q_{\mu}$ is finite and convex on $\mathbb{R}^{s}$ (cf. [14], [36]). Observe that, 
in general, an expected recourse function $Q_{\mu}$ may be nondifferentiable on a certain union of hyperplanes in $\mathbb{R}^{s}$ and that, indeed, differentiability properties of $Q_{\mu}$ depend on the degree of smoothness induced by the measure $\mu$ (cf. [14], [19], [35], [36] and Remark 4.8). Another observation shows that the uniqueness of solutions to (1.1) is guaranteed only if the constraint set $C$ picks just one element from the relevant level set of $g(\cdot)+Q_{\mu}(A \cdot)$. This set may be large since $Q_{\mu}(A \cdot)$ is constant on translates of the null space of the matrix $A$ (see Example 1.1 in [25]). Proposition 2.1 below provides some more insight into the structure of the solution set to (1.1) and elucidates the role of the set-valued mapping $\sigma(y):=\operatorname{argmin}\{g(x): x \in C, A x=y\}$ in this respect.

Note that assumption (A1) could be relaxed by introducting the set $\mathcal{K}=\left\{y \in \mathbb{R}^{s}\right.$ : $\left.Q_{\mu}(y)<+\infty\right\}$. Then (A2) and (A3) imply that $\mathcal{K}$ is a closed convex polyhedron and that $Q_{\mu}$ is convex and continuous on $\mathcal{K}$ (cf. [36]). Now (A1) can be replaced by the condition $\mathcal{K} \supseteq A(C)$ (relatively complete recourse), and much of the work done in this paper carries over to this more general setting by using spaces of functions defined on $\mathcal{K}$ instead of $\mathbb{R}^{s}$.

Let $K_{C}$ denote the set of all convex functions on $\mathbb{R}^{s}$ which forms a convex cone in the space $C^{0}\left(\mathbb{R}^{s}\right)$ of all continuous functions on $\mathbb{R}^{s}$. $K_{C}$ will serve as the set of possible perturbations of the given expected recourse function $Q_{\mu} \in K_{C}$. We define

$$
\begin{aligned}
& \varphi(Q):=\inf \{g(x)+Q(A x): x \in C\}, \\
& \psi(Q):=\operatorname{argmin}\{g(x)+Q(A x): x \in C\}
\end{aligned}
$$

and regard $\varphi$ and $\psi$ as mappings from $K_{C}$ into the extended reals and the set of all closed convex subsets of $\mathbb{R}^{m}$, respectively.

In this paper we develop a sensitivity analysis for the mappings $\varphi$ and $\psi$ at some given function $Q_{\mu}$. The stochastic programming origin of the model (1.1) takes a back seat and our results are stated in terms of general conditions on $Q_{\mu}$ and its perturbations $Q$. We identify conditions such that the value function $\varphi$ has first- and second-order directional derivatives and the solution-set mapping $\psi$ is directionally differentiable at $Q_{\mu}$ into admissible directions. Here, admissibility means that the direction belongs to the radial tangent cone to $K_{C}$ at $Q_{\mu}$, i.e.,

$$
T^{r}\left(K_{C} ; Q_{\mu}\right)=\left\{\lambda\left(Q-Q_{\mu}\right): Q \in K_{C}, \lambda>0\right\},
$$

ensuring that the difference quotients are well-defined. For $v$ belonging to $T^{r}\left(K_{C} ; Q_{\mu}\right)$ the Gateaux directional derivatives of $\varphi$ and $\psi$ at $Q_{\mu}$ and $\left(Q_{\mu}, \bar{x}\right), \bar{x} \in \psi\left(Q_{\mu}\right)$, respectively, are defined as

$$
\begin{aligned}
\varphi^{\prime}\left(Q_{\mu} ; v\right) & =\lim _{t \rightarrow 0+} \frac{1}{t}\left(\varphi\left(Q_{\mu}+t v\right)-\varphi\left(Q_{\mu}\right)\right), \\
\varphi^{\prime \prime}\left(Q_{\mu} ; v\right) & =\lim _{t \rightarrow 0+} \frac{1}{t^{2}}\left(\varphi\left(Q_{\mu}+t v\right)-\varphi\left(Q_{\mu}\right)-t \varphi^{\prime}\left(Q_{\mu} ; v\right)\right), \\
\psi^{\prime}\left(Q_{\mu}, \bar{x} ; v\right) & =\lim _{t \rightarrow 0+} \frac{1}{t}\left(\psi\left(Q_{\mu}+t v\right)-\bar{x}\right),
\end{aligned}
$$

if the limits exist. The third limit is understood in the sense of (Painlevé-Kuratowski) set convergence (e.g. [2]). Recall that the lower and upper set limits of a family $\left(S_{t}\right)_{t>0}$ 
of subsets of a metric space $(X, d)$ are defined as

$$
\begin{aligned}
\liminf _{t \rightarrow 0+} S_{t} & =\left\{x \in X: \lim _{t \rightarrow 0+} d\left(x, S_{t}\right)=0\right\}, \\
\limsup _{t \rightarrow 0+} S_{t} & =\left\{x \in X: \liminf _{t \rightarrow 0+} d\left(x, S_{t}\right)=0\right\} .
\end{aligned}
$$

Both sets are closed and the lower set limit is contained in the upper limit. If both limits coincide, the family $\left(S_{t}\right)_{t>0}$ is said to converge and its limit set is denoted by $\lim _{t \rightarrow 0+} S_{t}$. For sequences of sets $\left(S_{n}\right)_{n \in \mathbb{N}}$ the definitions of set limits are modified correspondingly. We also derive conditions implying that the limits defining the directional derivatives exist uniformly with respect to directions $v$ belonging to compact subsets of certain functional spaces. The limits are then called (first- or second-order) Hadamard directional derivatives and semiderivatives for set-valued maps, respectively. The corresponding directional derivatives are defined on tangent cones to the cone of convex functions in certain functional spaces. For more information on concepts of directional differentiability and multifunction differentiability we refer to [5], [30] and to [2], [4], [21], [23], respectively.

Let us fix some notations used throughout the paper. $\|\cdot\|$ and $\langle\cdot, \cdot\rangle$ denote the norm and scalar product, respectively, in some Euclidean space $\mathbb{R}^{n} ; B(x, r)$ denotes the open ball around $x \in \mathbb{R}^{n}$ with radius $r>0 ; d(x, D)$ denotes the distance of $x \in \mathbb{R}^{n}$ to the set $D \subseteq \mathbb{R}^{n}$; for a real-valued function $f$ on $\mathbb{R}^{n}, \nabla f$ denotes its gradient in $\mathbb{R}^{n}$ and the $(n, n)$-matrix $\nabla^{2} f$ its Hessian; if $f$ is locally Lipschitzian near $x \in \mathbb{R}^{n}, \partial f(x)$ denotes the Clarke subdifferential of $f$ at $x ; f^{\prime}(x ; d)$ denotes the directional derivative of $f$ at $x$ in direction $d$ if it exists; for $x \in C, T(C ; x)$ denotes the tangent cone to $C$ at $x$, i.e., $T(C ; x)=\liminf _{t \rightarrow 0+} \frac{1}{t}(C-x)=\operatorname{cl}\{\lambda(y-x): y \in C, \lambda>0\}$, where cl stands for closure; for $x \in C, \xi \in T(C ; x), T^{2}(C ; x, \xi)$ denotes the second order tangent set to $C$ at $x$ in direction $\xi$, i.e., $T^{2}(C ; x, \xi)=\liminf _{t \rightarrow 0+} \frac{1}{t^{2}}(C-x-t \xi)$ (note that $T^{2}(C ; x, \xi)$ is closed and convex; see [10] for further properties).

In our paper, we use the following linear metric spaces of real-valued functions on $\mathbb{R}^{s}$ : The space $C^{0}\left(\mathbb{R}^{s}\right)$ of continuous functions on $\mathbb{R}^{s}$ equipped with the distance

$d_{\infty}(f, \tilde{f})=\sum_{n=1}^{\infty} 2^{-n} \frac{\|f-\tilde{f}\|_{\infty, n}}{1+\|f-\tilde{f}\|_{\infty, n}}$, where

$\|f\|_{\infty, r}=\max _{\|y\| \leq r}|f(y)|$, for $f, \tilde{f} \in C^{0}\left(\mathbb{R}^{s}\right)$ and $r>0$

the space $C^{0,1}\left(\mathbb{R}^{s}\right)$ of locally Lipschitzian functions on $\mathbb{R}^{s}$ with the metric

$$
\begin{aligned}
d_{L}(f, \tilde{f}) & =\sum_{n=1}^{\infty} 2^{-n} \frac{\|f-\tilde{f}\|_{\infty, n}+\|f-\tilde{f}\|_{L, n}}{1+\|f-\tilde{f}\|_{\infty, n}+\|f-\tilde{f}\|_{L, n}}, \text { where } \\
\|f\|_{L, r} & =\sup \left\{\frac{|f(y)-f(\tilde{y})|}{\|y-\tilde{y}\|}:\|y\| \leq r,\|\tilde{y}\| \leq r, y \neq \tilde{y}\right\}, \\
& =\sup \{\|z\|: z \in \partial f(y),\|y\| \leq r\}, \text { for } f, \tilde{f} \in C^{0,1}\left(\mathbb{R}^{s}\right) \text { and } r>0 ;
\end{aligned}
$$

the space $C^{1}\left(\mathbb{R}^{s}\right)$ of continuously differentiable functions on $\mathbb{R}^{s}$ with the metric $d(f, \tilde{f})=$ $d_{\infty}(f, \tilde{f})+d_{\infty}(\nabla f, \nabla \tilde{f}), f, \tilde{f} \in C^{1}\left(\mathbb{R}^{s}\right)$, and the space $C^{1,1}\left(\mathbb{R}^{s}\right)$ of functions in $C^{1}\left(\mathbb{R}^{s}\right)$ 
whose gradients are locally Lipschitzian on $\mathbb{R}^{s}$ equipped with the distance $d(f, \tilde{f})=$ $d_{\infty}(f, \tilde{f})+d_{\infty}(\nabla f, \nabla \tilde{f})+d_{L}(\nabla f, \nabla \tilde{f})$ for all $f \in C^{1,1}\left(\mathbb{R}^{s}\right)$.

The sensitivity analysis of the mappings $\varphi$ and $\psi$ is carried out by exploiting structural properties of the optimization model (1.1). We obtain novel differentiability properties of solution sets and extend our earlier results on directional differentiability of optimal values in [12] considerably. As one might expect, the basic ingredients of our analysis are a Lipschitz continuity result for solution sets with respect to the distance in $C^{0,1}\left(\mathbb{R}^{s}\right)$ (Theorem 2.3) and a quadratic growth condition near solution sets (Theorem 2.6). Both theorems extend earlier results in [25] to more general situations for the first stage costs $g$ and constraint set $C$. All results in the paper apply to the linear-quadratic case, i.e., to linear or convex quadratic $g$ and polyhedral $C$. Indeed, all results are formulated as general as possible and most of them are accompanied by illustrative examples. The second-order analysis of $\varphi$ in Section 3 utilizes some ideas from [28] and [29], but its proof is entirely different and its Gateaux differentiability part is valid for nondifferentiable directions (Theorem 3.4). It is also elaborated that the Hadamard directional differentiability properties require the $C^{0}$-topology for the first-order result and the $C^{1}$-topology for the second-order one (Theorem 3.8), while the $C^{1,1}$-topology is needed for the semidifferentiability of the solution-set mapping $\psi$ (Theorem 4.7). All results on differentiability properties of $\psi$ in Section 4 are new and do not follow from recent sensitivity results (as e.g. [3], [6], [7], [16], [29]; see also the survey [8] for further references).

The results of Sections 3 and 4 have direct implications to asymptotic properties of values and solution sets of two-stage stochastic programs when applying nonparametric estimation procedures to approximate $Q_{\mu}$. For a discussion of some of the related aspects we refer to [11], where the delta-method is utilized and a central limit theorem for all selections belonging to a Castaing representation of the approximate solution sets is derived. Further applications to asymptotics are beyond the scope of this paper and will be done elsewhere.

\section{Basic directional properties}

The first step in our analysis of directional properties consists in establishing results on the lower Lipschitz continuity of $\psi$ and on the directional uniform quadratic growth of the objective near its solution set. Both results become important for our method of deriving directional differentiability properties for the optimal value function $\varphi$ and the solution set mapping $\psi$ at some given expected recourse function $Q_{\mu}$. Their proofs are based on a decomposition of the program

$$
\min \{g(x)+Q(A x): x \in C\},
$$

with $Q$ belonging to $K_{C}$, into two auxiliary problems. The first one is a convex program with decisions taken from $A(C)$ and the second represents a parametric convex program which does not depend on $Q$. 
Proposition 2.1 Let $Q \in K_{C}$ and $\psi(Q)$ be nonempty. Then we have

$$
\begin{aligned}
\varphi(Q) & =\inf \{\pi(y)+Q(y): y \in A(C)\}=\pi(A x)+Q(A x), \text { for any } x \in \psi(Q), \text { and } \\
\psi(Q) & =\sigma(Y(Q)), \text { where } \\
Y(Q) & :=\operatorname{argmin}\{\pi(y)+Q(y): y \in A(C)\}, \\
\pi(y) & :=\inf \{g(x): x \in C, A x=y\}, \text { and } \\
\sigma(y) & :=\operatorname{argmin}\{g(x): x \in C, A x=y\} \quad(y \in A(C)) .
\end{aligned}
$$

Moreover, $\pi$ is convex on $A(C)$ and $\operatorname{dom} \sigma$ is nonempty.

Proof. Let $\bar{x} \in \psi(Q)$. Then we have

$$
\varphi(Q)=g(\bar{x})+Q(A \bar{x}) \geq \pi(A \bar{x})+Q(A \bar{x}) \geq \inf \{\pi(y)+Q(y): y \in A(C)\} .
$$

For the converse inequality, let $\varepsilon>0$ and $\bar{y} \in A(C)$ be such that

$$
\pi(\bar{y})+Q(\bar{y}) \leq \inf \{\pi(y)+Q(y): y \in A(C)\}+\frac{\varepsilon}{2} .
$$

Then there exists a $\bar{x} \in C$ such that $A \bar{x}=\bar{y}$ and $g(\bar{x}) \leq \pi(\bar{y})+\frac{\varepsilon}{2}$. Hence,

$$
\begin{aligned}
\varphi(Q) & \leq g(\bar{x})+Q(A \bar{x}) \leq \pi(\bar{y})+Q(\bar{y})+\frac{\varepsilon}{2} \\
& \leq \inf \{\pi(y)+Q(y): y \in A(C)\}+\varepsilon
\end{aligned}
$$

Since $\varepsilon>0$ is arbitrary, the first statement has been shown. In particular, $x \in \sigma(A x)$ and $A x \in Y(Q)$ for any $x \in \psi(Q)$. Hence, it holds that $\psi(Q) \subseteq \sigma(Y(Q))$. Conversely, let $x \in \sigma(Y(Q))$. Then $x \in \sigma(y)$ for some $y \in Y(Q)$. Thus $A x=y$ and $g(x)=\pi(y)=$ $\pi(A x)$ implying

$$
\begin{aligned}
g(x)+Q(A x) & =\pi(A x)+Q(A x)=\inf \{\pi(y)+Q(y): y \in A(C)\} \\
& =\varphi(Q) \text { and } \quad x \in \psi(Q) .
\end{aligned}
$$

Since the convexity of $\pi$ is immediate, the proof is complete.

In the following, it will turn out that Lipschitzian properties of the solution set mapping $y \mapsto \sigma(y)$ and a quadratic growth property of $g$ near $\sigma(y)$ are essential. For the linearquadratic case we are in a comfortable situation in this respect. Namely, we have the following

Proposition 2.2 Let $g$ be linear or convex quadratic, $C$ be convex polyhedral and assume dom $\sigma$ to be nonempty. Then $\sigma$ is a polyhedral multifunction which is Hausdorff Lipschitzian on its domain $\operatorname{dom} \sigma=A(C)$, i.e., there exists a constant $L>0$ such that

$$
d_{H}(\sigma(y), \sigma(\tilde{y})) \leq L\|y-\tilde{y}\|, \quad \text { for all } y, \tilde{y} \in A(C),
$$

where $d_{H}$ denotes the (extended) Hausdorff distance on subsets of $\mathbb{R}^{m}$.

Moreover, for each $r>0$ there exists a constant $\eta(r)>0$ such that

$$
g(x) \geq \pi(A x)+\eta(r) d(x, \sigma(A x))^{2}, \quad \text { for all } \quad x \in C \cap B(0, r) .
$$

(Here $\pi$ and $\sigma$ are defined as in Proposition 2.1). 
Proof. The Lipschitz property of $\sigma$ is shown in [18], Theorem 4.2. To prove the second statement, let $g$ be of the form $g(x)=\langle H x, x\rangle+\langle c, x\rangle$, where $H$ is symmetric, positive semidefinite and $c \in \mathbb{R}^{m}$. For each $y \in A(C)$ we fix some $z(y) \in \sigma(y)$. An elementary characterization of solution sets to convex quadratic programs with linear constraints yields that

$$
\sigma(y)=\{x \in C: A x=y, H x=H z(y),\langle c, x\rangle=\langle c, z(y)\rangle\} .
$$

Due to the Lipschitz behaviour of convex polyhedra (cf. [34]), there exists a constant $L_{\sigma}>0$ such that

$$
d(x, \sigma(y)) \leq L_{\sigma}(\|H x-H z(y)\|+|\langle c, x\rangle-\langle c, z(y)\rangle|),
$$

for all $y \in A(C)$ and $x \in C$ with $A x=y$. Using the decomposition $H=H^{\frac{1}{2}} H^{\frac{1}{2}}$, where $H^{\frac{1}{2}}$ denotes the square root of $H$, and the representation $\langle c, x\rangle-\langle c, z(y)\rangle=$ $g(x)-\pi(y)-\left\|H^{\frac{1}{2}} x\right\|^{2}+\left\|H^{\frac{1}{2}} z(y)\right\|^{2}$, one arrives at the estimate

$$
d(x, \sigma(y)) \leq L_{\sigma}\left(\left\|H^{\frac{1}{2}}\right\|(1+\|x\|+\|z(y)\|)\left\|H^{\frac{1}{2}}(x-z(y))\right\|+g(x)-\pi(y)\right)
$$

for all $y \in A(C)$ and $x \in C$ with $A x=y$.

Now, let $r>0$ and let us fix some element $\bar{x} \in C \cap B(0, r)$ and a corresponding $z(A \bar{x}) \in \sigma(A \bar{x})$. For each $y \in A(C)$ we now select $z(y) \in \sigma(y)$ such that $\|z(y)-z(A \bar{x})\|=d(z(A \bar{x}), \sigma(y))$. Since $\sigma$ is Hausdorff Lipschitzian on $A(C)$, this implies $\|z(y)-z(A \bar{x})\| \leq L\|A \bar{x}-y\|$ for all $y \in A(C)$. Hence, there exists a constant $K(r)>0$ such that $\|z(A x)\| \leq K(r)$ for all $x \in C \cap B(0, r)$. Thus our estimate continues to $d(x, \sigma(A x))^{2} \leq \hat{L}(r)\left(\left\|H^{\frac{1}{2}}(x-z(A x))\right\|^{2}+(g(x)-\pi(A x))^{2}\right)$ for all $x \in C \cap B(0, r)$ and some constant $\hat{L}(r)>0$. Furthermore, the equation

$$
g\left(\frac{1}{2}(x+z(y))\right)=\frac{1}{2} g(x)+\frac{1}{2} g(z(y))-\frac{1}{4}\left\|H^{\frac{1}{2}}(x-z(y))\right\|^{2}
$$

implies $\left\|H^{\frac{1}{2}}(x-z(y))\right\|^{2} \leq 2(g(x)-\pi(y))$, for all $y \in A(C), x \in C$ with $A x=y$. Therefore, we finally obtain

$$
\begin{aligned}
d(x, \sigma(A x))^{2} & \leq \hat{L}(r)\left(2(g(x)-\pi(A x))+(g(x)-\pi(A x))^{2}\right) \\
& \leq \hat{L}(r) \max \{2, K(r)\}(g(x)-\pi(A x))
\end{aligned}
$$

for all $x \in C \cap B(0, r)$, where $K(r):=\sup _{x \in C \cap B(0, r)}(g(x)-\pi(A x))$.

Due to the above proposition, the main results in this section apply to the linearquadratic case. Although this case represents the main application of our results, the assumptions of the following theorems are formulated in terms of general conditions on the mapping $\sigma$ in order to gain generality and clarity. The first theorem states (lower) Lipschitz continuity of $\psi$ at $Q_{\mu}$ and supplements Theorem 2.4 in [25].

Theorem 2.3 Let $Q_{\mu} \in K_{C}, \psi\left(Q_{\mu}\right)$ be nonempty, bounded and $Q_{\mu}$ be strongly convex on some open, convex neighbourhood of $A \psi\left(Q_{\mu}\right)$. Let $\bar{x} \in \psi\left(Q_{\mu}\right)$ and assume that there exist a constant $L>0$ and a neighbourhood $U$ of $\bar{y}$ with $\{\bar{y}\}=A \psi\left(Q_{\mu}\right)$ such that

$$
d(\bar{x}, \sigma(y)) \leq L\|\bar{y}-y\|, \quad \text { for all } \quad y \in A(C) \cap U .
$$


Then there exist constants $\hat{L}>0, \delta>0$ and $r>0$ such that

$$
d(\bar{x}, \psi(Q)) \leq \hat{L}\left\|Q-Q_{\mu}\right\|_{L, r}
$$

whenever $Q \in K_{C}$ and $\left\|Q-Q_{\mu}\right\|_{L, r}<\delta$.

Proof. We may assume that $U$ is open, convex and that $Q_{\mu}$ is strongly convex on $U$. Let $V$ be an open, convex, bounded subset of $\mathbb{R}^{m}$ such that $\psi\left(Q_{\mu}\right) \subset V$ and $A(V) \subset U$. It follows from Proposition 2.3 in [25] (where a slightly different terminology is used) that there exists a constant $\delta>0$ such that $\emptyset \neq \psi(Q) \subset V$ whenever $Q \in K_{C}$ and

$$
\sup \left\{\|z\|: z \in \partial\left(Q-Q_{\mu}\right)(y), y \in \operatorname{cl} A(V)\right\}<\delta .
$$

Let $r>0$ be chosen such that $\operatorname{cl} A(V) \subset \bar{B}(0, r)$. Hence, we have $\emptyset \neq \psi(Q) \subset V$ whenever $Q \in K_{C},\left\|Q-Q_{\mu}\right\|_{L, r}<\delta$. Then Proposition 2.1 yields the relation $\psi(Q)=$ $\sigma(Y(q))$, where $Y(Q)=\operatorname{argmin}\{\pi(y)+Q(y): y \in A(C)\}$. Since $Q_{\mu}$ is strongly convex on $U$, there exists a constant $\kappa>0$ such that

$$
\kappa\|y-\bar{y}\|^{2} \leq \pi(y)+Q_{\mu}(y)-\left(\pi(\bar{y})+Q_{\mu}(\bar{y})\right), \quad \text { for all } \quad y \in U .
$$

Let $Q \in K_{C}$ with $\left\|Q-Q_{\mu}\right\|_{L, r}<\delta$ and let $\tilde{y} \in Y(Q)$. Since $y$ belongs to $A(V) \subset U$, we obtain

$$
\begin{aligned}
\kappa\|\tilde{y}-\bar{y}\|^{2} & \leq \pi(\tilde{y})+Q_{\mu}(\tilde{y})-\left(\pi(\bar{y})+Q_{\mu}(\bar{y})\right)+\pi(\bar{y})+Q(\bar{y})-(\pi(\tilde{y})+Q(\tilde{y})) \\
& =\left(Q-Q_{\mu}\right)(\bar{y})-\left(Q-Q_{\mu}\right)(\tilde{y})
\end{aligned}
$$

and, hence,

$$
\|\tilde{y}-\bar{y}\| \leq \frac{1}{\kappa} \frac{\left(Q-Q_{\mu}\right)(\bar{y})-\left(Q-Q_{\mu}\right)(\tilde{y})}{\|\bar{y}-\tilde{y}\|} \leq \frac{1}{\kappa}\left\|Q-Q_{\mu}\right\|_{L, r} .
$$

The proof can now be completed as follows. Let $Q \in K_{C}$ be such that $\left\|Q-Q_{\mu}\right\|_{L, r}<\delta$. Then

$$
\begin{aligned}
d(\bar{x}, \psi(Q)) & =d(\bar{x}, \sigma(Y(Q))) \leq \sup _{y \in Y(Q)} d(\bar{x}, \sigma(y)) \\
& \leq L \sup _{y \in Y(Q)}\|\bar{y}-y\| \leq \frac{L}{\kappa}\left\|Q-Q_{\mu}\right\|_{L, r} .
\end{aligned}
$$

Remark 2.4 The proof shows that a Lipschitz modulus of $\psi$ can be chosen as the quotient of a Lipschitz constant to $\sigma$ and a strong convexity constant to $Q_{\mu}$.

From the proof it is immediate that replacing the local Lipschitz condition on $\sigma$ by stronger conditions like

$$
\begin{aligned}
& \sup _{x \in \sigma(\bar{y})} d(x, \sigma(y)) \leq L\|\bar{y}-y\| \quad \text { or } \\
& d_{H}(\sigma(\bar{y}), \sigma(y)) \leq L\|\bar{y}-y\|, \quad \text { for all } y \in A(C) \cap U,
\end{aligned}
$$

leads to corresponding stronger Lipschitz continuity properties of solution sets. Because of Proposition 2.2, all of this applies to the linear-quadratic case. However, it is worth 
mentioning that the theorem also applies to more general problems such that the corresponding solution sets $\sigma(y)$ enjoy Lipschitzian properties. Conditions ensuring Lipschitz behaviour of $\sigma$ can be derived from stability results for the corresponding parametric generalized equation

$$
0 \in \nabla L(x, \lambda ; y)+N_{C \times \mathbb{R}^{s}}(x, \lambda)
$$

which describes the first order necessary optimality condition. Here $L(x, \lambda ; y):=g(x)+$ $\lambda^{T}(A x-y)$ is the Lagrangian function, $\nabla L(x, \lambda ; y)=\left(\begin{array}{c}\nabla g(x)+A^{T} \lambda \\ A x-y\end{array}\right)$, where $g$ is assumed to be continuously differentiable, and $N_{C \times \mathbb{R}^{s}}$ is the normal cone map of convex analysis. Such stability results are presently available for broad classes of parametric generalized equations (e.g. [16], [20], [22]). A typical recent result in this direction, which applies to our situation for twice continuously differentiable $g$, is Theorem 5.1 in [20]. It says that the solution set mapping of the parametric generalized equation (2.2) is pseudo-Lipschitzian around $(\bar{x}, \bar{\lambda} ; \bar{y})$ if the adjoint generalized equation

$$
0 \in \nabla^{2} L(\bar{x}, \bar{\lambda} ; \bar{y}) w^{*}+D^{*} N_{C \times \mathbb{R}^{s}}(\bar{x}, \bar{\lambda} ;-\nabla L(\bar{x}, \bar{\lambda} ; \bar{y}))\left(w^{*}\right)
$$

has only the trivial solution $w^{*}=0$.

Here $D^{*} N_{C \times \mathbb{R}^{s}}(\bar{x}, \bar{\lambda} ;-\nabla L(\bar{x}, \bar{\lambda} ; \bar{y}))$ is the Mordukhovich coderivative ([20]) of the normal cone multifunction at the point $(\bar{x}, \bar{\lambda} ;-\nabla L(\bar{x}, \bar{\lambda} ; \bar{y}))$ belonging to the graph of $N_{C \times \mathbb{R}^{s}}$. Translating this into our framework, we obtain that the mapping $\sigma$ is pseudoLipschitzian around $(\bar{x}, \bar{y})$ if the following two conditions are satisfied:

(a) There exists an element $\hat{x}$ belonging to the relative interior of $C$ such that $A \hat{x}=\bar{y}$ (Slater condition);

(b) the equations $A w_{1}^{*}=0$ and $0 \in \nabla^{2} g(\bar{x}) w_{1}^{*}+A^{T} w_{2}^{*}+D^{*} N_{C}(\bar{x}, \bar{\lambda} ;-\nabla g(\bar{x})-$ $\left.A^{T} \bar{\lambda}\right)\left(w_{1}^{*}\right)$ have only the trivial solution $w_{1}^{*}=0, w_{2}^{*}=0$. (Here $(\bar{x}, \bar{\lambda})$ is a solution of $(2.2)$ for $y=\bar{y}$.)

The next example shows that the theorem also applies to instances of two-stage stochastic programs with nonpolyhedral convex constraint sets $C$.

Example 2.5 In (1.1) - (1.3) let $m=2, s=1, g(x) \equiv 0, A=(1,0), q=(1,1)$, $W=(1,-1), \mu$ be the uniform distribution on $\left[-\frac{1}{2}, \frac{1}{2}\right]$ and $C=\left\{\left(x_{1}, x_{2}\right) \in \mathbb{R}^{2}\right.$ : $\left.x_{2}^{2} \leq x_{1}\right\}$. Then we have $\tilde{Q}(t)=|t|, Q_{\mu}(y)=\int_{\mathbb{R}}|\zeta-y| \mu(\mathrm{d} \zeta)=\left\{\begin{array}{ll}y^{2}+\frac{3}{4}, & y \in\left[-\frac{1}{2}, \frac{1}{2}\right] \\ |y| & \text { otherwise }\end{array}\right.$, $\psi\left(Q_{\mu}\right)=\{(0,0)\}$ and $Q_{\mu}$ is strongly convex on $\left(-\frac{1}{2}, \frac{1}{2}\right)$. For $y \in A(C)=\mathbb{R}_{+}$we have

$$
\sigma(y)=\{x \in C: A x=y\}=\left\{\left(y, x_{2}\right) \in \mathbb{R}^{2}: x_{2}^{2} \leq y\right\}=\{y\} \times[-\sqrt{y}, \sqrt{y}]
$$

and, hence $d((0,0), \sigma(y))=y$ for all $y \in \mathbb{R}_{+}$. Thus Theorem 2.3 applies for $\bar{x}=(0,0)$.

Example 2.8 shows that Theorem 2.3 gets lost if $Q_{\mu}$ fails to be strongly convex on some neighbourhood of $A \psi\left(Q_{\mu}\right)$. Our next result establishes a sufficient condition for the uniform quadratic growth near solution sets. 
Theorem 2.6 Let $Q_{\mu} \in K_{C}, \psi\left(Q_{\mu}\right)$ be nonempty, bounded and $Q_{\mu}$ be strongly convex on some open convex neighbourhood $U$ of $A \psi\left(Q_{\mu}\right)$. Assume that there exists a constant $L>0$ such that

$$
d_{H}(\sigma(y), \sigma(\tilde{y})) \leq L\|y-\tilde{y}\|, \quad \text { for all } \quad y, \tilde{y} \in A(C),
$$

and, for each $r>0$ there exists a constant $\eta(r)>0$ such that

$$
g(x) \geq \pi(A x)+\eta(r) d(x, \sigma(A x))^{2}, \quad \text { for all } x \in C \cap B(0, r) .
$$

Then, for some open, bounded neighbourhood $V$ of $\psi\left(Q_{\mu}\right)$ and each $v \in T^{r}\left(K_{C} ; Q_{\mu}\right)$, there exist constants $c>0$ and $\delta>0$ such that the following uniform growth condition holds:

$$
g(x)+\left(Q_{\mu}+t v\right)(A x) \geq \varphi\left(Q_{\mu}+t v\right)+c d\left(x, \psi\left(Q_{\mu}+t v\right)\right)^{2},
$$

for all $x \in C \cap V$ and $t \in[0, \delta)$.

Proof. Let $v \in T^{r}\left(K_{C}, Q_{\mu}\right)$ and $V$ be an open, bounded subset of $\mathbb{R}^{m}$ such that $\psi\left(Q_{\mu}\right) \subset V$ and $A(V) \subseteq U$. As in Theorem 2.3 we choose $\delta>0$ such that $\emptyset \neq$ $\psi\left(Q_{\mu}+t v\right) \subset V$ and, in addition, that $Q_{\mu}+t v$ is strongly convex on $U$ for all $t \in[0, \delta)$ (with a uniform constant $\kappa>0$ ). For each $t \in[0, \delta)$ Proposition 2.1 then yields that $\psi\left(Q_{\mu}+t v\right)=\sigma\left(y_{t}\right)$, where $y_{t}$ is the unique minimizer of the strongly convex function $\pi+Q_{\mu}+t v$ on $A(C)$ and, moreover, we have $\kappa\left\|y-y_{t}\right\|^{2} \leq \pi(y)+\left(Q_{\mu}+t v\right)(y)-\varphi\left(Q_{\mu}+t v\right)$, for all $y \in A(C) \cap U$. Now, we choose $r>0$ such that $V \subseteq B(0, r)$ and continue for each $x \in C \cap V$ and $t \in[0, \delta)$ as follows:

$$
\begin{aligned}
d\left(x, \psi\left(Q_{\mu}+t v\right)\right)^{2} & =d\left(x, \sigma\left(y_{t}\right)\right)^{2} \\
& \leq 2\left(d(x, \sigma(A x))^{2}+d_{H}\left(\sigma(A x), \sigma\left(y_{t}\right)\right)^{2}\right) \\
& \leq 2\left(\frac{1}{\eta(r)}(g(x)-\pi(A x))+L^{2}\left\|A x-y_{t}\right\|^{2}\right) \\
& \leq 2\left(\frac{1}{\eta(r)}(g(x)-\pi(A x))+\frac{L^{2}}{\kappa}\left(\pi(A x)+\left(Q_{\mu}+t v\right)(A x)-\varphi\left(Q_{\mu}+t v\right)\right)\right) \\
& \leq 2 \max \left\{\frac{1}{\eta(r)}, \frac{L^{2}}{\kappa}\right\}\left(g(x)+\left(Q_{\mu}+t v\right)(A x)-\varphi\left(Q_{\mu}+t v\right)\right)
\end{aligned}
$$

Putting $c^{-1}=2 \max \left\{\frac{1}{\eta(r)}, \frac{L^{2}}{\kappa}\right\}$ completes the proof.

The following examples show that the quadratic growth condition gets lost even for the original problem, i.e. $t=0$, if either the Lipschitz condition for $\sigma$ or the strong convexity property for $Q_{\mu}$ are violated.

Example 2.7 Consider again the set-up of Example 2.5. It holds that $d_{H}(\sigma(y), \sigma(0))=$ $\left(y^{2}+y\right)^{\frac{1}{2}}$, for all $y \in \mathbb{R}_{+}=A(C)$, and $\sigma$ is not Hausdorff Lipschitzian on $A(C)$.

Supposed there exists a neighbourhood $V$ of $\psi\left(Q_{\mu}\right)=\{(0,0)\}$ and a constant $\varrho>0$ such that the growth condition

$$
\varrho d\left(x, \psi\left(Q_{\mu}\right)\right)^{2}=\varrho\|x\|^{2} \leq Q_{\mu}\left(x_{1}\right)-\varphi\left(Q_{\mu}\right)=x_{1}^{2}, \quad \text { for all } \quad x \in C \cap V,
$$

is satisfied. Since the sequence $\left(\left(\frac{1}{n}, \frac{1}{\sqrt{n}}\right)\right)$ belongs to $C \cap V$ for sufficiently large $n \in \mathbb{N}$, this would imply $\varrho\left(\frac{1}{n^{2}}+\frac{1}{n}\right) \leq \frac{1}{n^{2}}$ for large $n$, which is a contradiction. 
Example 2.8 In (1.1) - (1.3) let $m=s=1, g(x) \equiv 0, A=1, C=\mathbb{R}, q=(1,1)$, $W=(1,-1)$ and $\mu$ be the probability distribution on $\mathbb{R}$ having the density

$$
f_{\mu}(z)=\left\{\begin{array}{cl}
|z|, & z \in[-1,1] \\
0 & \text { otherwise }
\end{array}\right.
$$

Then $Q_{\mu}(y)=\int_{I R}|\zeta-y| \mu(\mathrm{d} \zeta)=\left\{\begin{array}{ll}\frac{1}{3}|y|^{3}+\frac{2}{3}, & y \in[-1,1] \\ |y| & \text { otherwise }\end{array}\right.$,

$\psi\left(Q_{\mu}\right)=\{0\}$, and there is no neighbourhood of $\psi\left(Q_{\mu}\right)$ where $Q_{\mu}$ is strongly convex.

It is clear that the quadratic growth condition fails to hold, since the inequality $\varrho x^{2} \leq$ $Q_{\mu}(x)-\varphi\left(Q_{\mu}\right)=\frac{1}{3}|x|^{3}$ cannot be true for some $\varrho>0$ and all $x$ belonging to some neighbourhood of $x=0$.

With the linear function $v(x)=-x(x \in \mathbb{R})$ we obtain for all $t \in[0,1]$ that $\psi\left(Q_{\mu}+t v\right)=$ $\{\sqrt{t}\}$ (cf. Example 3.7). Hence, the lower Lipschitz property of $\psi$ has got lost, too.

Since the strong convexity and later also the strict convexity of the expected recourse function $Q_{\mu}$ (on certain convex subsets of $\mathbb{R}^{s}$ ) form essential conditions in most of our results, we record a theorem (Theorem 2.2 in [27]) that provides a handy criterion to check these properties for problem $(1.1)-(1.3)$.

Proposition 2.9 Let $V \subset \mathbb{R}^{s}$ be open convex and assume (A1), (A3). Consider the following conditions:

$(\mathrm{A} 2)^{*} \quad$ int $M_{D}=\left\{t \in \mathbb{R}^{s}: W^{T} t<q\right\} \neq \emptyset ;$

(A4) $\mu$ is absolutely continuous on $\mathbb{R}^{s}$;

$(\mathrm{A} 4)^{*} \quad \mu$ satisfies (A4) and there exist a density $f_{\mu}$ for $\mu$ and a constant

$\delta>0$ such that $f_{\mu}(z) \geq \delta$ whenever $d(z, V) \leq \delta$.

Then (A2)* and (A4) imply that $Q_{\mu}$ is strictly convex on $V$ if $V$ is a subset of the support of $\mu$, and (A2)*, (A4)* imply that $Q_{\mu}$ is strongly convex on $V$.

In addition, it is shown in [27] that under (A1) - (A4) the condition (A2)* is also necessary for the strict convexity of $Q_{\mu}$. For extended simple recourse models (i.e. $W=(H,-H)$ with some nonsingular $(s, s)$-matrix $H)(\mathrm{A} 2)^{*}$ is equivalent to $q^{+}+q^{-}>0$ (componentwise), where $q=\left(q^{+}, q^{-}\right)$and $q^{+}, q^{-} \in \mathbb{R}^{s}$. This may be used to check strict or strong convexity properties in the Examples 2.5 and 2.8.

\section{Directional derivatives of optimal values}

In this section, we study first- and second-order directional differentiability properties of the optimal value function $\varphi$ on its domain $K_{C}$. We begin with the first-order analysis and show that $\varphi$ as a mapping from $K_{C}$ to the extended reals is Hadamard directionally differentiable at some given expected recourse function $Q_{\mu} \in K_{C}$. Here $K_{C}$ is regarded as a subset of $C^{0}\left(\mathbb{R}^{s}\right)$. Recall that $\varphi$ is Hadamard directionally differentiable at $Q_{\mu}$ on 
$K_{C}$ iff for all sequences $\left(v_{n}\right)$ converging to some $v$ in $C^{0}\left(\mathbb{R}^{s}\right)$ and all sequences $t_{n} \rightarrow 0+$ such that the elements $Q_{\mu}+t_{n} v_{n}$ belong to $K_{C}$ the limit

$$
\varphi^{\prime}\left(Q_{\mu} ; v\right)=\lim _{n \rightarrow \infty} \frac{1}{t_{n}}\left(\varphi\left(Q_{\mu}+t_{n} v_{n}\right)-\varphi\left(Q_{\mu}\right)\right)
$$

exists. Since the condition $Q_{\mu}+t_{n} v_{n} \in K_{C}$ means that $v_{n}=\frac{1}{t_{n}}\left(Q_{n}-Q_{\mu}\right)$ for some $Q_{n} \in K_{C}$, the limit $v$ belongs to the tangent cone $T\left(K_{C} ; Q_{\mu}\right)$ to $K_{C}$ at $Q_{\mu}$ in $C^{0}\left(\mathbb{R}^{s}\right)$. In [32], [33] this property is also called Hadamard directional differentiability tangentially to $K_{C}$.

Proposition 3.1 Let $Q_{\mu} \in K_{C}$ and assume that $\psi\left(Q_{\mu}\right)$ is nonempty, bounded. Then $\varphi$ is Hadamard directionally differentiable at $Q_{\mu}$ on $K_{C}$ and it holds for all $v \in$ $T\left(K_{C} ; Q_{\mu}\right)$,

$$
\varphi^{\prime}\left(Q_{\mu} ; v\right)=\min \left\{v(A x): x \in \psi\left(Q_{\mu}\right)\right\} .
$$

If, in addition, $Q_{\mu}$ is strictly convex on some open convex neighbourhood of $A \psi\left(Q_{\mu}\right)$, we have

$$
\varphi^{\prime}\left(Q_{\mu} ; v\right)=v(\bar{y}), \quad \text { where } \quad\{\bar{y}\}=A \psi\left(Q_{\mu}\right) .
$$

Proof. Arguing similarly as in the proof of Propostion 2.1 in [24] there exists a neighbourhood $\mathcal{N}$ of $Q_{\mu}$ in $C^{0}\left(\mathbb{R}^{s}\right)$ such that $\psi(Q)$ is nonempty for all $Q \in K_{C} \cap \mathcal{N}$. Let $\left(t_{n}\right)$ and $\left(v_{n}\right)$ be sequences such that $t_{n} \rightarrow 0+, v_{n} \rightarrow v$ in $C^{0}\left(\mathbb{R}^{s}\right)$ and $Q_{\mu}+t_{n} v_{n}$ belongs to $K_{C}$ for all $n \in \mathbb{N}$. Then $Q_{\mu}+t_{n} v_{n} \in K_{C} \cap \mathcal{N}$ for sufficiently large $n \in I N$. Let $x_{n} \in \psi\left(Q_{\mu}+t_{n} v_{n}\right)$ for those $n \in \mathbb{N}$. Since $\psi$ is Berge upper semicontinuous at $Q_{\mu}$ $([24])$, the sequence $\left(x_{n}\right)$ has an accumulation point $x \in \psi\left(Q_{\mu}\right)$ and we obtain

$$
\begin{aligned}
& \limsup _{n \rightarrow \infty} \frac{1}{t_{n}}\left(\varphi\left(Q_{\mu}+t_{n} v_{n}\right)-\varphi\left(Q_{\mu}\right)\right) \\
\geq & \limsup _{n \rightarrow \infty} \frac{1}{t_{n}}\left(g\left(x_{n}\right)+\left(Q_{\mu}+t_{n} v_{n}\right)\left(A x_{n}\right)-g\left(x_{n}\right)-Q_{\mu}\left(A x_{n}\right)\right) \\
= & \limsup _{n \rightarrow \infty} v_{n}\left(A x_{n}\right) \geq v(A x),
\end{aligned}
$$

where the last inequality follows from the uniform convergence of $\left(v_{n}\right)$ to $v$ on bounded subsets of $\mathbb{R}^{s}$. In order to show the reverse inequality for lim inf, let $x \in \psi\left(Q_{\mu}\right)$. Then

$$
\begin{aligned}
& \liminf _{n \rightarrow \infty} \frac{1}{t_{n}}\left(\varphi\left(Q_{\mu}+t_{n} v_{n}\right)-\varphi\left(Q_{\mu}\right)\right) \\
\leq & \liminf _{n \rightarrow \infty} \frac{1}{t_{n}}\left(g(x)+\left(Q_{\mu}+t_{n} v_{n}\right)(A x)-g(x)-Q_{\mu}(A x)\right) \\
= & \liminf _{n \rightarrow \infty} v_{n}(A x)=v(A x) .
\end{aligned}
$$

This completes the proof of the first part. The second part is an immediate conclusion, since $A \psi\left(Q_{\mu}\right)$ is a singleton whenever $Q_{\mu}$ is strictly convex on some of its open, convex neighbourhoods. 
The preceding result can also be proved by using the methodology of Theorem 6.4.1 in [26]. There the compactness of the constraint set is assumed and Gateaux directional differentiability of $\varphi$ at $Q_{\mu}$ together with its Lipschitz continuity is shown. Here we prefer a direct two-sided argument, which will also be used in the subsequent secondorder analysis of $\varphi$. Namely, we will first derive an upper bound for the second-order Hadamard directional derivative of $\varphi$ at some $Q_{\mu} \in K_{C}$, where $K_{C}$ is equipped with the $C^{0,1}$-topology. Secondly, we identify conditions implying that the upper bound coincides with the Gateaux directional derivative of $\varphi$ at $Q_{\mu}$ for all directions taken from $T^{r}\left(K_{C} ; Q_{\mu}\right)$.

Lemma 3.2 Let $y \in \mathbb{R}^{s}, Q_{\mu} \in K_{C}, t_{n} \rightarrow 0+,\left(Q_{n}\right)$ be a sequence in $K_{C}$ such that $v_{n}:=\frac{1}{t_{n}}\left(Q_{n}-Q_{\mu}\right) \rightarrow v$ in $C^{0,1}\left(\mathbb{R}^{s}\right)$ and let $\left(\xi_{n}\right)$ be a sequence converging to $\xi$ in $\mathbb{R}^{s}$. Then we have $\limsup _{n \rightarrow \infty} \frac{1}{t_{n}}\left(v_{n}\left(y+t_{n} \xi_{n}\right)-v_{n}(y)\right) \leq \max _{\zeta \in \partial v(y)}\langle\zeta, \xi\rangle$.

Proof. Each function $v_{n}$ is locally Lipschitzian on $\mathbb{R}^{s}$ and, hence, Lebourg's mean value theorem for Clarke's subdifferential ([9]) implies the existence of elements $\tilde{y}_{n}$ belonging to the segments $\left[y, y+t_{n} \xi_{n}\right]$ such that

$$
\frac{1}{t_{n}}\left(v_{n}\left(y+t_{n} \xi_{n}\right)-v_{n}(y)\right) \in\left\{\left\langle\zeta, \xi_{n}\right\rangle: \zeta \in \partial v_{n}\left(\tilde{y}_{n}\right)\right\}
$$

The convergence $v_{n} \rightarrow v$ in $C^{0,1}\left(\mathbb{R}^{s}\right)$ implies that

$$
\sup \left\{\|\zeta\|: \zeta \in \partial\left(v_{n}-v\right)(y),\|y\| \leq r\right\} \underset{n \rightarrow \infty}{\longrightarrow} 0
$$

holds for any $r>0$. This yields

$$
d_{H}\left(\partial v_{n}\left(\tilde{y}_{n}\right), \partial v\left(\tilde{y}_{n}\right)\right) \leq \sup \left\{\|\zeta\|: \zeta \in \partial\left(v_{n}-v\right)\left(\tilde{y}_{n}\right)\right\} \underset{n \rightarrow \infty}{\longrightarrow} 0 .
$$

Here $d_{H}$ denotes the Hausdorff distance and the inequality is a consequence of general properties of the subdifferential (cf. Lemma 2.1 in [25]). Hence, there exist elements $\widetilde{\zeta}_{n}$ belonging to $\partial v\left(\tilde{y}_{n}\right)$ such that

$$
\frac{1}{t_{n}}\left(v_{n}\left(y+t_{n} \xi_{n}\right)-v_{n}(y)\right) \leq\left\|\xi_{n}\right\| d_{H}\left(\partial v_{n}\left(\tilde{y}_{n}\right), \partial v\left(\tilde{y}_{n}\right)\right)+\left\langle\tilde{\zeta}_{n}, \xi_{n}\right\rangle
$$

and, for some $\tilde{\zeta} \in \partial v(y)$,

$$
\begin{aligned}
\limsup _{n \rightarrow \infty} \frac{1}{t_{n}}\left(v_{n}\left(y+t_{n} \xi_{n}\right)-v_{n}(y)\right) & \leq \limsup _{n \rightarrow \infty}\left\langle\tilde{\zeta}_{n}, \xi_{n}\right\rangle=\langle\tilde{\zeta}, \xi\rangle \\
& \leq \max _{\zeta \in \partial v(y)}\langle\zeta, \xi\rangle .
\end{aligned}
$$

Here, the identity follows from the upper semicontinuity of $\partial v(\cdot)$. This completes the proof.

Proposition 3.3 Let $Q_{\mu} \in K_{C}$ and assume that $\psi\left(Q_{\mu}\right)$ is nonempty, bounded. Let $g$ be twice continuously differentiable, $Q_{\mu}$ be strictly convex on some open convex neighbourhood of $A \psi\left(Q_{\mu}\right)$ and twice continuously differentiable at $\bar{y}$, where $\{\bar{y}\}=A \psi\left(Q_{\mu}\right)$. 
Let $\bar{x} \in \psi\left(Q_{\mu}\right), t_{n} \rightarrow 0+$ and $\left(Q_{n}\right)$ be a sequence in $K_{C}$ such that $v_{n}:=\frac{1}{t_{n}}\left(Q_{n}-Q_{\mu}\right) \rightarrow v$ in $C^{0,1}\left(\mathbb{R}^{s}\right)$. Then

$$
\begin{aligned}
\limsup _{n \rightarrow \infty} & \frac{1}{t_{n}^{2}}\left(\varphi\left(Q_{\mu}+t_{n} v_{n}\right)-\varphi\left(Q_{\mu}\right)-t_{n} \varphi^{\prime}\left(Q_{\mu} ; v_{n}\right)\right) \\
\leq \inf & \left\{\langle\nabla g(\bar{x}), z\rangle+\left\langle\nabla Q_{\mu}(\bar{y}), A z\right\rangle+\frac{1}{2}\left\langle\nabla^{2} g(\bar{x}), \xi, \xi\right\rangle\right. \\
+ & \left.\frac{1}{2}\left\langle\nabla^{2} Q_{\mu}(\bar{y}) A \xi, A \xi\right\rangle+\max _{\zeta \in \partial v(\bar{y})}\langle\zeta, A \xi\rangle: \xi \in S(\bar{x}), z \in T^{2}(C ; \bar{x}, \xi)\right\},
\end{aligned}
$$

where $S(\bar{x}):=\left\{\xi \in T(C ; \bar{x}):\langle\nabla g(\bar{x}), \xi\rangle+\left\langle\nabla Q_{\mu}(\bar{y}), A \xi\right\rangle=0\right\}, T(C ; \bar{x})$ is the tangent cone to $C$ at $\bar{x}$ and $T^{2}(C ; \bar{x}, \xi)$ the second order tangent set to $C$ at $\bar{x}$ in direction $\xi$.

Proof. Let $\xi \in S(\bar{x})$ and $z \in T^{2}(C ; \bar{x}, \xi)$. Then there exists a sequence $\left(z_{n}\right)$ such that $z_{n} \rightarrow z$ and $\bar{x}+t_{n} \xi++t_{n}^{2} z_{n} \in C$ for all $n \in I N$. Using Proposition 3.1, this allows for the following estimate

$$
\begin{aligned}
& \varphi\left(Q_{\mu}+t_{n} v_{n}\right)-\varphi\left(Q_{\mu}\right)-t_{n} \varphi^{\prime}\left(Q_{\mu} ; v_{n}\right) \\
\leq & g\left(\bar{x}+t_{n} \xi+t_{n}^{2} z_{n}\right)+Q_{\mu}\left(A\left(\bar{x}+t_{n} \xi+t_{n}^{2} z_{n}\right)\right)+t_{n} v_{n}\left(A\left(\bar{x}+t_{n} \xi+t_{n}^{2} z_{n}\right)\right) \\
& \quad-g(\bar{x})-Q_{\mu}(A \bar{x})-t_{n} v_{n}(A \bar{x}) \\
= & {\left[g\left(\bar{x}+t_{n} \xi+t_{n}^{2} z_{n}\right)-g(\bar{x})-t_{n}\langle\nabla g(\bar{x}), \xi\rangle\right] } \\
& +\left[Q_{\mu}\left(A\left(\bar{x}+t_{n} \xi+t_{n}^{2} z_{n}\right)\right)-Q_{\mu}(A \bar{x})-t_{n}\left\langle\nabla Q_{\mu}(A \bar{x}), A \xi\right\rangle\right] \\
& +t_{n}\left[v_{n}\left(A\left(\bar{x}+t_{n} \xi+t_{n}^{2} z_{n}\right)\right)-v_{n}(A \bar{x})\right] .
\end{aligned}
$$

After dividing by $t_{n}^{2}$ and using Lemma 3.2 the limes superior as $n \rightarrow \infty$ of the righthand side can be bounded above by

$$
\begin{aligned}
\langle\nabla g(\bar{x}), z\rangle+\frac{1}{2}\left\langle\nabla^{2} g(\bar{x}) \xi, \xi\right\rangle & +\left\langle\nabla Q_{\mu}(A \bar{x}), A z\right\rangle \\
& +\frac{1}{2}\left\langle\nabla^{2} Q_{\mu}(A \bar{x}) A \xi, A \xi\right\rangle+\max _{\zeta \in \partial v(A \bar{x})}\langle\zeta, A \xi\rangle .
\end{aligned}
$$

Taking the infimum on the right-hand side yields the assertion.

We notice that the upper second-order Hadamard directional derivative $\limsup _{n \rightarrow \infty} \frac{1}{t_{n}^{2}}\left(\varphi\left(Q_{\mu}+t_{n} v_{n}\right)-\varphi\left(Q_{\mu}\right)-t_{n} \varphi^{\prime}\left(Q_{\mu} ; v_{n}\right)\right)$ is nonpositive, since $\varphi$ is concave on $K_{C}$ and, hence, the inequality $\varphi\left(Q_{\mu}+t_{n} v_{n}\right)-\varphi\left(Q_{\mu}\right)=\varphi\left(Q_{n}\right)-\varphi\left(Q_{\mu}\right) \leq \varphi^{\prime}\left(Q_{\mu} ; Q_{n}-Q_{\mu}\right)=$ $t_{n} \varphi^{\prime}\left(Q_{\mu} ; v_{n}\right)$ is valid. We also note that the upper bound is nonpositve, since $(0,0)$ belongs to $S(\bar{x}) \times T^{2}(C ; \bar{x}, 0)=S(\bar{x}) \times T(C ; \bar{x})$.

Next we consider particular perturbations $Q_{n}$ of $Q_{\mu}$, namely, $Q_{n}:=Q_{\mu}+\lambda t_{n}\left(Q-Q_{\mu}\right)$ for some $Q \in K_{C}, \lambda>0$ and sufficiently large $n \in \mathbb{N}$. Then $v_{n}=\lambda\left(Q-Q_{\mu}\right) \in$ $T^{r}\left(K_{C} ; Q_{\mu}\right)$. In the following result we give conditions implying that the second-order (Gateaux) directional derivative exists and coincides with the upper bound of the previous proposition. The result extends those in [12] although its proof parallels in parts that of Theorem 3.6 in [12]. 
Theorem 3.4 Let $Q_{\mu} \in K_{C}$ and assume that $\psi\left(Q_{\mu}\right)$ is nonempty, bounded. Let $g$ be twice continuously differentiable, $Q_{\mu}$ be strictly convex on some open convex neighbourhood of $A \psi\left(Q_{\mu}\right)$ and twice continuously differentiable at $\bar{y}$, where $\{\bar{y}\}=A \psi\left(Q_{\mu}\right)$. Let $\bar{x} \in \psi\left(Q_{\mu}\right), v \in T^{r}\left(K_{C} ; Q_{\mu}\right)$ and assume that

(i) $d\left(\bar{x}, \psi\left(Q_{\mu}+t v\right)\right)=O(t)$ for small $t>0$, and

(ii) the second-order set $S^{2}(\bar{x}, \xi):=\left\{z \in T^{2}(C ; \bar{x}, \xi):\langle\nabla g(\bar{x}), z\rangle+\left\langle\nabla Q_{\mu}(\bar{y}), A z\right\rangle=0\right\}$ is nonempty for each $\xi \in S(\bar{x})$.

Then the second-order Gateaux directional derivative of $\varphi$ at $Q_{\mu}$ in direction $v$ exists and it holds that

$$
\begin{aligned}
\varphi^{\prime \prime}\left(Q_{\mu} ; v\right) & =\lim _{t \rightarrow 0+} \frac{1}{t^{2}}\left(\varphi\left(Q_{\mu}+t_{n} v\right)-\varphi\left(Q_{\mu}\right)-t \varphi^{\prime}\left(Q_{\mu} ; v\right)\right) \\
& =\inf \left\{\frac{1}{2}\left\langle\nabla^{2} g(\bar{x}) \xi, \xi\right\rangle+\frac{1}{2}\left\langle\nabla^{2} Q_{\mu}(\bar{y}) A \xi, A \xi\right\rangle+v^{\prime}(\bar{y} ; A \xi): \xi \in S(\bar{x})\right\} .
\end{aligned}
$$

Moreover, the infimum is attained at some $\bar{\xi} \in S(\bar{x})$ having the property that $\varphi^{\prime \prime}\left(Q_{\mu} ; v\right)=\frac{1}{2} v^{\prime}(\bar{y} ; A \bar{\xi})$.

(Here $S(\bar{x})$ and $T^{2}(C ; \bar{x}, \xi)$ are defined as in the previous result, $v^{\prime}(\bar{y} ; \eta)$ is the directional derivative of $v$ at $\bar{y}$ in direction $\eta$ and $O(t)$ denotes a real quantity such that $\frac{1}{t}|O(t)|$ is bounded as $t \rightarrow 0+$.)

Proof. (i) implies that there exist constants $L>0, \delta>0$ and elements $x(t) \in$ $\psi\left(Q_{\mu}+t v\right)$ such that $\|x(t)-\bar{x}\| \leq L t$ for all $t \in(0, \delta)$. By expanding $g$ and $Q_{\mu}$ and using Proposition 3.1 we obtain

$$
\begin{aligned}
& \varphi\left(Q_{\mu}+t v\right)-\varphi\left(Q_{\mu}\right)-t \varphi^{\prime}\left(Q_{\mu} ; v\right) \\
= & g(x(t))+Q_{\mu}(A x(t))+t v(A x(t))-g(\bar{x})-Q_{\mu}(A \bar{x})-t v(A \bar{x}) \\
= & \langle\nabla g(\bar{x}), x(t)-\bar{x}\rangle+\frac{1}{2}\left\langle\nabla^{2} g(\bar{x})(x(t)-\bar{x}), x(t)-\bar{x}\right\rangle \\
& +\left\langle\nabla Q_{\mu}(A \bar{x}), A(x(t)-\bar{x})\right\rangle+\frac{1}{2}\left\langle\nabla^{2} Q_{\mu}(A \bar{x})(A(x(t)-\bar{x})), A(x(t)-\bar{x})\right\rangle \\
& +t(v(A x(t))-v(A \bar{x}))+o\left(\|x(t)-\bar{x}\|^{2}\right) .
\end{aligned}
$$

Moreover, we have that $o\left(\|x(t)-\bar{x}\|^{2}\right)=o\left(t^{2}\right)$ where $o(s)$ denotes a real quantity having the property $\frac{1}{s} o(s) \rightarrow 0$ as $s \rightarrow 0+$. Since the optimality of $\bar{x}$ implies

$$
\langle\nabla g(\bar{x}), x(t)-\bar{x}\rangle+\left\langle\nabla Q_{\mu}(A \bar{x}), A(x(t)-\bar{x})\right\rangle \geq 0
$$

for any $t \in(0, \delta)$, we have

$$
\begin{aligned}
& \frac{1}{t^{2}}\left(\varphi\left(Q_{\mu}+t v\right)-\varphi\left(Q_{\mu}\right)-t \varphi^{\prime}\left(Q_{\mu} ; v\right)\right) \\
\geq & \frac{1}{2}\left\langle\nabla^{2} g(\bar{x}) \frac{1}{t}(x(t)-\bar{x}), \frac{1}{t}(x(t)-\bar{x})\right\rangle \\
& +\frac{1}{2}\left\langle\nabla^{2} Q_{\mu}(\bar{y}) A\left(\frac{1}{t}(x(t)-\bar{x})\right), A\left(\frac{1}{t}(x(t)-\bar{x})\right)\right\rangle \\
& +\frac{1}{t}\left(v\left(A \bar{x}+t A\left(\frac{1}{t}(x(t)-\bar{x})\right)\right)-v(A \bar{x})\right)+o(1) .
\end{aligned}
$$


Now take a sequence $\left(t_{n}\right)$ tending to $0+$ in such a way that

$$
\begin{aligned}
& \liminf _{t \rightarrow 0+} \frac{1}{t^{2}}\left(\varphi\left(Q_{\mu}+t v\right)-\varphi\left(Q_{\mu}\right)-t \varphi^{\prime}\left(Q_{\mu} ; v\right)\right) \\
& =\lim _{n \rightarrow \infty} \frac{1}{t_{n}^{2}}\left(\varphi\left(Q_{\mu}+t_{n} v\right)-\varphi\left(Q_{\mu}\right)-t_{n} \varphi^{\prime}\left(Q_{\mu} ; v\right)\right)
\end{aligned}
$$

and that $\xi_{n}:=\frac{1}{t_{n}}\left(x\left(t_{n}\right)-\bar{x}\right) \underset{n \rightarrow \infty}{\longrightarrow} \bar{\xi}$. The latter is possible since $\left\|\frac{1}{t_{n}}\left(x\left(t_{n}\right)-\bar{x}\right)\right\| \leq L$ for $n \in I N$ sufficiently large. Then $\bar{\xi} \in T(C ; \bar{x})$ and Proposition 3.1 yields

$$
\begin{aligned}
v(\bar{y}) & =\varphi^{\prime}\left(Q_{\mu} ; v\right)=\lim _{n \rightarrow \infty} \frac{1}{t_{n}}\left(\varphi\left(Q_{\mu}+t_{n} v\right)-\varphi\left(Q_{\mu}\right)\right) \\
& =\lim _{n \rightarrow \infty} \frac{1}{t_{n}}\left(g\left(\bar{x}+t_{n} \xi_{n}\right)+\left(Q_{\mu}+t_{n} v\right)\left(A\left(\bar{x}+t_{n} \xi_{n}\right)\right)-g(\bar{x})-Q_{\mu}(A \bar{x})\right) \\
& =\langle\nabla g(\bar{x}), \bar{\xi}\rangle+\left\langle\nabla Q_{\mu}(A \bar{x}), A \bar{\xi}\right\rangle+v(A \bar{x}) .
\end{aligned}
$$

This implies $\bar{\xi} \in S(\bar{x})$. From (3.1) and (3.2) we obtain

$$
\begin{aligned}
& \liminf _{t \rightarrow 0+} \frac{1}{t^{2}}\left(\varphi\left(Q_{\mu}+t v\right)-\varphi\left(Q_{\mu}\right)-t \varphi^{\prime}\left(Q_{\mu} ; v\right)\right) \\
\geq & \lim _{n \rightarrow \infty}\left\{\frac{1}{2}\left\langle\nabla^{2} g(\bar{x}) \xi_{n}, \xi_{n}\right\rangle+\frac{1}{2}\left\langle\nabla^{2} Q_{\mu}(\bar{y}) A \xi_{n}, A \xi_{n}\right\rangle+\frac{1}{t_{n}}\left(v\left(\bar{y}+t_{n} A \xi_{n}\right)-v(\bar{y})\right)\right\} \\
= & \frac{1}{2}\left\langle\nabla^{2} g(\bar{x}) \bar{\xi}, \bar{\xi}\right\rangle+\frac{1}{2}\left\langle\nabla^{2} Q_{\mu}(\bar{y}) A \bar{\xi}, A \bar{\xi}\right\rangle+v^{\prime}(\bar{y} ; A \bar{\xi}) .
\end{aligned}
$$

Here we have used the fact that $v$ is Hadamard directionally differentiable and Clarke regular $([9])$, i.e. $v^{\prime}(\bar{y} ; \eta)=\max _{\zeta \in \partial v(\bar{y})}\langle\zeta, \eta\rangle$. From Proposition 3.3 we obtain

$$
\begin{aligned}
& \limsup _{t \rightarrow 0+} \frac{1}{t^{2}}\left(\varphi\left(Q_{\mu}+t v\right)-\varphi\left(Q_{\mu}\right)-t \varphi^{\prime}\left(Q_{\mu} ; v\right)\right) \\
& \leq \inf \left\{\left\langle\nabla g(\bar{x}, z\rangle+\left\langle\nabla Q_{\mu}(\bar{y}), A z\right\rangle+\frac{1}{2}\left\langle\nabla^{2} g(\bar{x}) \xi, \xi\right\rangle\right.\right. \\
&\left.+\frac{1}{2}\left\langle\nabla^{2} Q_{\mu}(\bar{y}) A \xi, A \xi\right\rangle+v^{\prime}(\bar{y} ; A \xi): \xi \in S(\bar{x}), z \in T^{2}(C ; \bar{x}, \xi)\right\} \\
&=\inf \left\{\frac{1}{2}\left\langle\nabla^{2} g(\bar{x}) \xi, \xi\right\rangle+\frac{1}{2}\left\langle\nabla^{2} Q_{\mu}(\bar{y}) A \xi, A \xi\right\rangle+v^{\prime}(\bar{y} ; A \xi): \xi \in S(\bar{x})\right\} .
\end{aligned}
$$

The latter equality is due to (ii) and to the fact that the necessary optimality condition for $\bar{x}$ yields

$$
\langle\nabla g(\bar{x}), z\rangle+\left\langle\nabla Q_{\mu}(\bar{y}), A z\right\rangle \geq 0, \text { for all } z \in T^{2}(C ; \bar{x}, \xi), \xi \in S(\bar{x}) .
$$

Hence, the limit $\lim _{t \rightarrow 0+} \frac{1}{t^{2}}\left(\varphi\left(Q_{\mu}+t v\right)-\varphi\left(Q_{\mu}\right)-t \varphi^{\prime}\left(Q_{\mu} ; v\right)\right)$ exists and is equal to the infimum subject to $\xi \in S(\bar{x})$. Moreover, this infimum is attained at $\bar{\xi} \in S(\bar{x})$. For the remainder of the proof we put $a(\xi):=v^{\prime}(\bar{y} ; A \xi)$ and

$$
B(\xi):=\frac{1}{2}\left\langle\nabla^{2} g(\bar{x}) \xi, \xi\right\rangle+\frac{1}{2}\left\langle\nabla^{2} Q_{\mu}(\bar{y}) A \xi, A \xi\right\rangle, \quad \text { for all } \xi \in \mathbb{R}^{n} .
$$


Since $S(\bar{x})$ is a (convex) cone, we have $S(\bar{x})=\lambda S(\bar{x})$ for any $\lambda>0$ and thus

$$
\begin{aligned}
0 \leq f(\lambda) & :=B(\lambda \bar{\xi})+a(\lambda \bar{\xi})-B(\bar{\xi})-a(\bar{\xi}) \\
& =\lambda^{2} B(\bar{\xi})+\lambda a(\bar{\xi})-(B(\bar{\xi})+a(\bar{\xi})), \text { for all } \lambda>0 .
\end{aligned}
$$

In case of $B(\bar{\xi})>0$, the quadratic function $f$ vanishes at $\lambda=1$ with the property $f^{\prime}(1)=2 B(\bar{\xi})+a(\bar{\xi})=0$ and the final assertion is shown. If $B(\bar{\xi})=0$, the fact that $0 \leq f(\lambda)=\lambda a(\bar{\xi})-a(\bar{\xi})$ holds for any $\lambda>0$, implies $a(\bar{\xi})=0$. Thus $\varphi^{\prime \prime}\left(Q_{\mu} ; v\right)=0=$ $\frac{1}{2} a(\bar{\xi})$ and the proof is complete.

The proof shows that the previous theorem remains true when replacing condition (ii) by the condition that both infima in (3.3) coincide. Next we state a more handy criterion implying that $\varphi^{\prime \prime}\left(Q_{\mu} ; v\right)$ exists for any direction $v \in T^{r}\left(K_{C} ; Q_{\mu}\right)$.

Corollary 3.5 Let $Q_{\mu} \in K_{C}$ and assume that $\psi\left(Q_{\mu}\right)$ is nonempty, bounded. Let $g$ be twice continuously differentiable, $Q_{\mu}$ be strongly convex on some open convex neighbourhood of $A \psi\left(Q_{\mu}\right)$ and twice continuously differentiable at $\bar{y}$ where $\{\bar{y}\}=A \psi\left(Q_{\mu}\right)$. Let $\bar{x} \in \psi\left(Q_{\mu}\right)$ and assume that

(i)' there exist a constant $L>0$ and a neighbourhood $U$ of $\bar{y}$ such that $d(\bar{x}, \sigma(y)) \leq L\|\bar{y}-y\|$ for all $y \in A(C) \cap U$, where $\sigma(y):=\operatorname{argmin}\{g(x): x \in C, A x=y\}, y \in A(C)$,

(ii) the second-order set $S^{2}(\bar{x}, \xi)=\left\{z \in T^{2}(C ; \bar{x}, \xi):\langle\nabla g(\bar{x}), z\rangle+\left\langle\nabla Q_{\mu}(\bar{y}), A z\right\rangle=0\right\}$ is nonempty for each $\xi \in S(\bar{x})$.

Then the second-order Gateaux directional derivative of $\varphi$ at $Q_{\mu}$ exists for any direction $v \in T^{r}\left(K_{C} ; Q_{\mu}\right)$ and the formula for $\varphi^{\prime \prime}\left(Q_{\mu} ; v\right)$ in Theorem 3.4 holds true.

Moreover, condition (ii) is satisfied if $C$ is polyhedral and (i)' is satisfied for any $\bar{x} \in$ $\psi\left(Q_{\mu}\right)$ if, in addition to the polyhedrality of $C, g$ is linear or (convex) quadratic.

Proof. Let $v \in T^{r}\left(K_{C} ; Q_{\mu}\right)$. Theorem 2.3 then says that there exist constants $\hat{L}>0$, $\delta>0, r>0$ such that

$$
d\left(\bar{x}, \psi\left(Q_{\mu}+t v\right)\right) \leq \hat{L}\|v\|_{L, r} t \quad \text { whenever } \quad\|v\|_{L, r} t<\delta .
$$

Hence, the strong convexity of $Q_{\mu}$ and condition (i)' imply that condition (i) of the previous theorem is satisfied and that the first part of the assertion is shown. If $C$ is polyhedral, we have $T^{2}(C ; \bar{x}, \xi)=T(T(C ; \bar{x}) ; \xi)$ and thus $0 \in T^{2}(C ; \bar{x}, \xi)$ for any $\xi \in T(C ; \bar{x})$. Hence, (ii) is satisfied. If $C$ is polyhedral and $g$ is linear or (convex) quadratic, Proposition 2.2 implies (i)' to hold for any $\bar{x} \in \psi\left(Q_{\mu}\right)=\sigma(\bar{y})$.

Let us consider two illustrative examples to provide some insight into the benefit and limits of the previous results.

Example 3.6 We revisit Example 2.5 and know that condition (i)' is satisfied for $\bar{x}=(0,0)$. Furthermore, it holds that $T(C ; \bar{x})=\mathbb{R}_{+} \times \mathbb{R}$ and

$$
T^{2}(C ; \bar{x}, \xi)=\left\{\begin{array}{ll}
\mathbb{R}^{2}, & \xi_{1}>0 \\
\left\{x_{1} \in \mathbb{R}: x_{1} \geq \xi_{2}^{2}\right\} \times \mathbb{R}, & \xi_{1}=0
\end{array} \text {, for any } \xi \in T(C ; \bar{x}) .\right.
$$


Hence, (ii) and the general assumptions of Corollary 3.5 are satisfied and $\varphi^{\prime \prime}\left(Q_{\mu} ; v\right)$ exists for any $v \in T^{r}\left(K_{C} ; Q_{\mu}\right)$. It holds that $\varphi^{\prime \prime}\left(Q_{\mu} ; v\right)=\frac{1}{2} v^{\prime}\left(0, \bar{\xi}_{1}\right)$, where $\bar{\xi}=\left(\bar{\xi}_{1}, \bar{\xi}_{2}\right) \in$ $\operatorname{argmin}\left\{\xi_{1}^{2}+v^{\prime}\left(0, \xi_{1}\right):\left(\xi_{1}, \xi_{2}\right) \in \mathbb{R}_{+} \times \mathbb{R}\right\}$. Let us finally replace the function $g(x) \equiv 0$ by $g(x)=x_{1}$. Then $\sigma(y)$ and $\psi\left(Q_{\mu}\right)$ remain unchanged, $S(\bar{x})=\{\xi \in T(C ; \bar{x})$ : $\langle\nabla g(\bar{x}), \xi\rangle=0\}=\{0\} \times \mathbb{R}$, and condition (ii) is violated. But, since we have

$$
\begin{aligned}
& \inf \left\{\langle\nabla g(\bar{x}), z\rangle+\left\langle\nabla Q_{\mu}(\bar{y}), A z\right\rangle+\frac{1}{2}\left\langle\nabla^{2} g(\bar{x}) \xi, \xi\right\rangle\right. \\
& \left.+\frac{1}{2}\left\langle\nabla^{2} Q_{\mu}(\bar{y}) A \xi, A \xi\right\rangle+v^{\prime}(\bar{y}, A \xi): \xi \in S(\bar{x}), z \in T^{2}(C ; \bar{x}, \xi)\right\} \\
= & \inf \left\{z_{1}+\xi_{1}^{2}+v^{\prime}\left(0, \xi_{1}\right): \xi \in S(\bar{x}), z \in T^{2}(C ; \bar{x}, \xi)\right\} \\
= & \inf \left\{z_{1}: z_{1} \geq \xi_{2}^{2}, \xi_{2} \in \mathbb{R}\right\}=0,
\end{aligned}
$$

both infima in (3.3) coincide, the result holds true and we have

$$
\varphi^{\prime \prime}\left(Q_{\mu} ; v\right)=\frac{1}{2} v^{\prime}(0 ; 0)=0 \text { for any } v \in T^{r}\left(K_{C} ; Q_{\mu}\right) .
$$

Example 3.7 Here we revisit Example 2.8, and have

$$
Q_{\mu}(y)=\frac{1}{3}|y|^{3}+\frac{2}{3}, \text { for all }|y| \leq 1, \text { and } \psi\left(Q_{\mu}\right)=\{0\}, \varphi\left(Q_{\mu}\right)=\frac{2}{3} .
$$

For the function $v(x)=-x(x \in \mathbb{R})$ and $t \in[0,1)$ we obtain

$$
\begin{aligned}
\varphi\left(Q_{\mu}+t v\right) & =\inf \left\{Q_{\mu}(x)-t x: x \in \mathbb{R}\right\}, \text { and } \\
\psi\left(Q_{\mu}+t v\right) & =\operatorname{argmin}\left\{Q_{\mu}(x)-t x: x \in \mathbb{R}\right\} \\
& =\left\{x \in \mathbb{R}: Q_{\mu}^{\prime}(x)=t, Q_{\mu}(x)-t x=\varphi\left(Q_{\mu}+t v\right)\right\}=\{\sqrt{t}\}, \\
\varphi\left(Q_{\mu}+t v\right) & =\frac{2}{3}\left(1-t^{\frac{3}{2}}\right) .
\end{aligned}
$$

Then $\varphi^{\prime}\left(Q_{\mu} ; v\right)=0$ and $\frac{1}{t^{2}}\left(\varphi\left(Q_{\mu}+t v\right)-\varphi\left(Q_{\mu}\right)-\varphi^{\prime}\left(Q_{\mu} ; v\right)\right)=-\frac{2}{3} t^{-\frac{1}{2}}$. Hence, $\varphi$ has no second-order directional derivative at $Q_{\mu}$ in direction $v$. Note that there is no neighbourhood of $\bar{x}=0$ where $Q_{\mu}$ is strongly convex.

Finally, we aim at showing that $\varphi$ is even second-order Hadamard directionally differentiable at $Q_{\mu}$ when equipping $K_{C}$ with a suitable topology. To this end we need a certain counterpart of Lemma 3.2 for the corresponding limes inferior. Since this is not available for nonsmooth functions, it is a natural idea to consider the space $C^{1}\left(\mathbb{R}^{s}\right)$, to restrict $\varphi$ to the subset $K_{C} \cap C^{1}$ and to equip $K_{C} \cap C^{1}$ with the $C^{1}$-topology. Then we are able to show that the assumptions of Corollary 3.5 even imply the second-order Hadamard directional differentiability of $\varphi$ at $Q_{\mu}$.

Theorem 3.8 Let $Q_{\mu} \in K_{C} \cap C^{1}$ and assume that $\psi\left(Q_{\mu}\right)$ is nonempty, bounded. Let $g$ be twice continuously differentiable, $Q_{\mu}$ be strongly convex on some open convex neighbourhood of $A \psi\left(Q_{\mu}\right)$ and twice continuously differentiable at $\bar{y}$ where $\{\bar{y}\}=A \psi\left(Q_{\mu}\right)$. Let $\bar{x} \in \psi\left(Q_{\mu}\right)$ and assume the conditions (i)' and (ii) of Corollary 3.5 to hold.

Then the second-order Hadamard directional derivative of $\varphi$ at $Q_{\mu}$ exists in any direction $v$ belonging to the tangent cone $T\left(K_{C} \cap C^{1} ; Q_{\mu}\right)$ in $C^{1}\left(\mathbb{R}^{s}\right)$, i.e., for any such 
$v$, and all sequences $t_{n} \rightarrow 0+$ and $\left(Q_{n}\right)$ in $K_{C}$ such that $v_{n}:=\frac{1}{t_{n}}\left(Q_{n}-Q_{\mu}\right) \rightarrow v$ in $C^{1}\left(\mathbb{R}^{s}\right)$ the limit

$$
\varphi^{\prime \prime}\left(Q_{\mu} ; v\right)=\lim _{n \rightarrow \infty} \frac{1}{t_{n}}\left(\varphi\left(Q_{\mu}+t_{n} v_{n}\right)-\varphi\left(Q_{\mu}\right)-t_{n} \varphi^{\prime}\left(Q_{\mu} ; v_{n}\right)\right)
$$

exists, and it holds

$$
\varphi^{\prime \prime}\left(Q_{\mu} ; v\right)=\inf \left\{\frac{1}{2}\left\langle\nabla^{2} g(\bar{x}) \xi, \xi\right\rangle+\frac{1}{2}\left\langle\nabla^{2} Q_{\mu}(\bar{y}) A \xi, A \xi\right\rangle+\langle\nabla v(\bar{y}), A \xi\rangle: \xi \in S(\bar{x})\right\}
$$

where $S(\bar{x})=\left\{\xi \in T(C ; \bar{x}):\langle\nabla g(\bar{x}), \xi\rangle+\left\langle\nabla Q_{\mu}(\bar{y}), A \xi\right\rangle=0\right\}$.

Proof. Let $v \in T\left(K_{C} \cap C^{1} ; Q_{\mu}\right), t_{n} \rightarrow 0+$ and $\left(Q_{n}\right)$ be a sequence in $K_{C}$ such that $v_{n}=\frac{1}{t_{n}}\left(Q_{n}-Q_{\mu}\right) \rightarrow v$ in $C^{1}\left(\mathbb{R}^{s}\right)$. Condition (i)' together with Theorem 2.3 then imply that there exist constants $L>0, r>0, n_{0} \in I N$ and elements $x_{n} \in \psi\left(Q_{\mu}+t_{n} v_{n}\right)$ such that

$$
\left\|x_{n}-\bar{x}\right\| \leq L t_{n}\left\|v_{n}\right\|_{L, r}, \quad \text { for all } n \in I N, n \geq n_{0}
$$

Since the sequence $\left(v_{n}\right)$ converges in $C^{1}\left(\mathbb{R}^{s}\right)$, the norms $\left\|v_{n}\right\|_{L, r}$ are uniformly bounded and we have $\left\|x_{n}-\bar{x}\right\|=O\left(t_{n}\right)$. Expanding $g$ and $Q_{\mu}$ as in the proof of Theorem 3.4 we obtain analogously to (3.1), for all $n \geq n_{0}$ :

$$
\begin{aligned}
& \frac{1}{t_{n}^{2}}\left(\varphi\left(Q_{\mu}+t_{n} v_{n}\right)-\varphi\left(Q_{\mu}\right)-t_{n} \varphi^{\prime}\left(Q_{\mu} ; v_{n}\right)\right) \\
\geq & \frac{1}{2}\left\langle\nabla^{2} g(\bar{x})\left(\frac{1}{t_{n}}\left(x_{n}-\bar{x}\right)\right), \frac{1}{t_{n}}\left(x_{n}-\bar{x}\right)\right\rangle \\
& +\frac{1}{2}\left\langle\nabla^{2} Q_{\mu}(\bar{y}) A\left(\frac{1}{t_{n}}\left(x_{n}-\bar{x}\right)\right), A\left(\frac{1}{t_{n}}\left(x_{n}-\bar{x}\right)\right)\right\rangle \\
& +\frac{1}{t_{n}}\left(v_{n}\left(\bar{y}+t_{n} A\left(\frac{1}{t_{n}}\left(x_{n}-\bar{x}\right)\right)\right)-v_{n}(\bar{y})\right)+o(1) .
\end{aligned}
$$

Putting $\xi_{n}:=\frac{1}{t_{n}}\left(x_{n}-\bar{x}\right)$ and using the mean value theorem for $v_{n}$ we may continue with some $\bar{y}_{n} \in\left[\bar{y}, \bar{y}+t_{n} A \xi_{n}\right]$ as follows:

$$
\begin{aligned}
& \frac{1}{t_{n}^{2}}\left(\varphi\left(Q_{\mu}+t_{n} v_{n}\right)-\varphi\left(Q_{\mu}\right)-t_{n} \varphi^{\prime}\left(Q_{\mu} ; v_{n}\right)\right) \\
\geq & \frac{1}{2}\left\langle\nabla^{2} g(\bar{x}) \xi_{n}, \xi_{n}\right\rangle+\frac{1}{2}\left\langle\nabla^{2} Q_{\mu}(\bar{y}) A \xi_{n}, A \xi_{n}\right\rangle+\left\langle\nabla v_{n}\left(\bar{y}_{n}\right), A \xi_{n}\right\rangle+o(1) .
\end{aligned}
$$

Arguing as in the proof of Theorem 3.4 and using $v_{n} \rightarrow v$ in $C^{1}\left(\mathbb{R}^{s}\right)$ we arrive at the estimate

$$
\begin{gathered}
\liminf _{n \rightarrow \infty} \frac{1}{t_{n}^{2}}\left(\varphi\left(Q_{\mu}+t_{n} v_{n}\right)-\varphi\left(Q_{\mu}\right)-t_{n} \varphi^{\prime}\left(Q_{\mu} ; v_{n}\right)\right) \\
\geq \frac{1}{2}\left\langle\nabla^{2} g(\bar{x}) \bar{\xi}, \bar{\xi}\right\rangle+\frac{1}{2}\left\langle\nabla^{2} Q_{\mu}(\bar{y}) A \bar{\xi}, A \bar{\xi}\right\rangle+\langle\nabla v(\bar{y}), A \bar{\xi}\rangle
\end{gathered}
$$


for some element $\bar{\xi} \in S(\bar{x})$.

Furthermore, we conclude from (ii) and Proposition 3.3 that

$$
\begin{aligned}
& \limsup _{n \rightarrow \infty} \frac{1}{t_{n}^{2}}\left(\varphi\left(Q_{\mu}+t_{n} v_{n}\right)-\varphi\left(Q_{\mu}\right)-t_{n} \varphi^{\prime}\left(Q_{\mu} ; v_{n}\right)\right) \\
\leq & \inf \left\{\frac{1}{2}\left\langle\nabla^{2} g(\bar{x}) \xi, \xi\right\rangle+\frac{1}{2}\left\langle\nabla^{2} Q_{\mu}(\bar{y}) A \xi, A \xi\right\rangle+\langle\nabla v(\bar{y}), A \xi\rangle: \xi \in S(\bar{x})\right\} .
\end{aligned}
$$

Hence, the desired limit exists and the proof is complete.

Let us finally note that all minimization problems appearing as bounds or formulas for second-order directional derivatives represent convex programs. Those in the results 3.4, 3.5 and 3.8 have convex cone constraints, which are polyhedral if $C$ is polyhedral. Moreover, the solution sets of the convex minimization problems in 3.4, 3.5 and 3.8 are nonempty. Indeed, we show next that these solution sets represent certain derivatives of the set-valued mapping $\psi$ at the pair $\left(Q_{\mu}, \bar{x}\right)$.

\section{Differentiability of solution sets}

It is well-known that second-order differentiability properties of optimal values in perturbed optimization are intrinsic for establishing the differentiability of solutions (see e.g. [8]). We also pursue this approach and derive conditions implying directional differentiability properties of the solution set mapping by exploiting the results of the previous section. Our first results in this direction concern Gateaux directional differentiability, and complement Theorem 3.4 and its corollary.

Theorem 4.1 Assume that the general conditions on $g, Q_{\mu}$ and $C$ of Theorem 3.4 are satisfied. Let $\bar{x} \in \psi\left(Q_{\mu}\right), v \in T^{r}\left(K_{C} ; Q_{\mu}\right)$ and suppose the conditions (i) and (ii) of Theorem 3.4 to be satisfied. In addition, assume that

(iii) there exist a neighbourhood $V$ of $\psi\left(Q_{\mu}\right)$ and constants $c>0, \delta>0$ such that the uniform growth condition

$$
g(x)+\left(Q_{\mu}+t v\right)(A x) \geq \varphi\left(Q_{\mu}+t v\right)+c d\left(x, \psi\left(Q_{\mu}+t v\right)\right)^{2}
$$

for all $x \in C \cap V$ and $t \in[0, \delta)$, is satisfied.

Then the Gateaux directional derivative of $\psi$ at the pair $\left(Q_{\mu}, \bar{x}\right)$ into direction $v$ exists and it holds that

$$
\begin{aligned}
& \psi^{\prime}\left(Q_{\mu}, \bar{x} ; v\right)=\lim _{t \rightarrow 0+} \frac{1}{t}\left(\psi\left(Q_{\mu}+t v\right)-\bar{x}\right) \\
= & \operatorname{argmin}\left\{\frac{1}{2}\left\langle\nabla^{2} g(\bar{x}) \xi, \xi\right\rangle+\frac{1}{2}\left\langle\nabla^{2} Q_{\mu}(\bar{y}) A \xi, A \xi\right\rangle+v^{\prime}(\bar{y} ; A \xi): \xi \in S(\bar{x})\right\} .
\end{aligned}
$$


Proof. Let $M(\bar{x} ; v)$ denote the solution set in the assertion. First we show that $\limsup _{t \rightarrow 0+} \frac{1}{t}\left(\psi\left(Q_{\mu}+t v\right)-\bar{x}\right) \subseteq M(\bar{x} ; v)$.

Let $\xi \in \limsup _{t \rightarrow 0+} \frac{1}{t}\left(\psi\left(Q_{\mu}+t v\right)-\bar{x}\right)$. Then there exists a sequence $\left(t_{n}, \xi_{n}\right)$ converging to $(0+, \xi)$ such that $\xi_{n} \in \frac{1}{t_{n}}\left(\psi\left(Q_{\mu}+t_{n} v\right)-\bar{x}\right)$ and, thus, $\bar{x}+t_{n} \xi_{n} \in \psi\left(Q_{\mu}+t_{n} v\right)$ for all $n \in \mathbb{N}$.

Hence, analogously to the proof of Theorem 3.4 we deduce that $\xi$ belongs to $S(\bar{x})$. In view of Theorem 3.4 it remains to show that $\frac{1}{2}\left\langle\nabla^{2} g(\bar{x}) \xi, \xi\right\rangle+\frac{1}{2}\left\langle\nabla^{2} Q_{\mu}(\bar{y}) A \xi, A \xi\right\rangle+$ $v^{\prime}(\bar{y} ; A \xi) \leq \varphi^{\prime \prime}\left(Q_{\mu} ; v\right)$. By expanding $g$ and $Q_{\mu}$ as in the proof of Theorem 3.4, we obtain analogously to $(3.1)$ :

$$
\begin{aligned}
& \varphi\left(Q_{\mu}+t_{n} v\right)-\varphi\left(Q_{\mu}\right)-t_{n} \varphi^{\prime}\left(Q_{\mu} ; v\right) \\
= & g\left(\bar{x}+t_{n} \xi_{n}\right)+Q_{\mu}\left(A\left(\bar{x}+t_{n} \xi_{n}\right)\right)-g(\bar{x})-Q_{\mu}(A \bar{x})+t_{n}\left(v\left(A\left(\bar{x}+t_{n} \xi_{n}\right)\right)-v(A \bar{x})\right) \\
\geq \frac{1}{2} t_{n}^{2}\left\langle\nabla^{2} g(\bar{x}) \xi_{n}, \xi_{n}\right\rangle & +\frac{1}{2} t_{n}^{2}\left\langle\nabla^{2} Q_{\mu}(\bar{y}) A \xi_{n}, A \xi_{n}\right\rangle \\
& \quad+t_{n}\left(v\left(A\left(\bar{x}+t_{n} \xi_{n}\right)\right)-v(A \bar{x})\right)+o\left(\left\|t_{n} \xi_{n}\right\|^{2}\right) .
\end{aligned}
$$

After dividing by $t_{n}^{2}$ and taking the $\lim _{n \rightarrow \infty}$ on both sides of the inequality, we obtain the desired estimate. In a second step we show that

$$
M(\bar{x} ; v) \subseteq \liminf _{t \rightarrow 0+} \frac{1}{t}\left(\psi\left(Q_{\mu}+t v\right)-\bar{x}\right)
$$

or, equivalently, that it holds for any $\xi \in M(\bar{x}, v)$,

$$
\lim _{t \rightarrow 0} \frac{1}{t} d\left(\bar{x}+t \xi, \psi\left(Q_{\mu}+t v\right)\right)=0 .
$$

Let $\xi \in M(\bar{x} ; v)$ and $\left(t_{n}\right)$ be a sequence with $t_{n} \rightarrow 0+$. We have to show that $\lim _{n \rightarrow \infty} \frac{1}{t_{n}} d\left(\bar{x}+t_{n} \xi, \psi\left(Q_{\mu}+t_{n} v\right)\right)=0$. Since $\xi \in S(\bar{x})$, there exists an element $z \in S^{2}(\bar{x}, \xi)$ and a sequence $\left(z_{n}\right)$ converging to $z$ with $\bar{x}+t_{n} \xi+t_{n}^{2} z_{n} \in C$ for all $n \in I N$. Hence, it suffices to show that

$$
\lim _{n \rightarrow \infty} \frac{1}{t_{n}} d\left(\bar{x}+t_{n} \xi+t_{n}^{2} z_{n}, \psi\left(Q_{\mu}+t_{n} v\right)\right)=0 .
$$

Condition (iii) implies the following estimate for all sufficiently large $n \in \mathbb{N}$ :

$$
\begin{aligned}
& c d\left(\bar{x}+t_{n} \xi+t_{n}^{2} z_{n}, \psi\left(Q_{\mu}+t_{n} v\right)\right)^{2} \\
\leq & g\left(\bar{x}+t_{n} \xi+t_{n}^{2} z_{n}\right)+\left(Q_{\mu}+t_{n} v\right)\left(A\left(\bar{x}+t_{n} \xi+t_{n}^{2} z_{n}\right)\right)-\varphi\left(Q_{\mu}+t_{n} v\right) .
\end{aligned}
$$

By expanding $g$ and $Q_{\mu}$ as in the proof of Theorem 3.4 and using the fact that $\xi$ belongs to $S(\bar{x})$, we may continue

$$
\begin{aligned}
= & t_{n}^{2}\left\langle\nabla g(\bar{x}), z_{n}\right\rangle+\frac{1}{2} t_{n}^{2}\left\langle\nabla^{2} g(\bar{x})\left(\xi+t_{n} z_{n}\right), \xi+t_{n} z_{n}\right\rangle \\
& +t_{n}^{2}\left\langle\nabla Q_{\mu}(\bar{y}), A z_{n}\right\rangle+\frac{1}{2} t_{n}^{2}\left\langle\nabla^{2} Q_{\mu}(\bar{y})\left(A\left(\xi+t_{n} z_{n}\right)\right), A\left(\xi+t_{n} z_{n}\right)\right\rangle \\
& -\left(\varphi\left(Q_{\mu}+t_{n} v\right)-\varphi\left(Q_{\mu}\right)-t_{n} \varphi^{\prime}\left(Q_{\mu} ; v\right)\right) \\
& +t_{n}\left(v\left(A\left(\bar{x}+t_{n} \xi+t_{n}^{2} z_{n}\right)\right)-v(A \bar{x})\right)+o\left(t_{n}^{2}\left\|\xi+t_{n} z_{n}\right\|^{2}\right) .
\end{aligned}
$$


After dividing by $t_{n}^{2}$ and taking the $\limsup _{n}$ on both sides of the latter inequality, we obtain

$$
\begin{aligned}
& \limsup _{n \rightarrow \infty} \frac{c}{t_{n}^{2}} d\left(\bar{x}+t_{n} \xi+t_{n}^{2} z_{n}, \psi\left(Q_{\mu}+t_{n} v\right)\right)^{2} \\
\leq & \langle\nabla g(\bar{x}), z\rangle+\left\langle\nabla Q_{\mu}(\bar{y}), A z\right\rangle+\frac{1}{2}\left\langle\nabla^{2} g(\bar{x}) \xi, \xi\right\rangle \\
& +\frac{1}{2}\left\langle\nabla^{2} Q_{\mu}(\bar{y}) A \xi, A \xi\right\rangle-\varphi^{\prime \prime}\left(Q_{\mu} ; v\right)+v^{\prime}(\bar{y} ; A \xi)=0,
\end{aligned}
$$

where we made use of $z \in S^{2}(\bar{x}, \xi), \xi \in M(\bar{x} ; v)$ and Theorem 3.4. This completes the proof.

Complementing Corollary 3.5 we provide a result on the directional differentiability of $\psi$ at $Q_{\mu}$ into any direction $v \in T^{r}\left(K_{C} ; Q_{\mu}\right)$.

Theorem 4.2 Assume that the general conditions on $g, Q_{\mu}$ and $C$ of Corollary 3.5 are satisfied. Let $\bar{x} \in \psi\left(Q_{\mu}\right)$ and assume that

(i)" there exists a constant $L>0$ such that

$$
d_{H}(\sigma(y), \sigma(\tilde{y})) \leq L\|y-\tilde{y}\|, \quad \text { for all } y, \tilde{y} \in A(C)
$$

and, for each $r>0$, there exists a constant $\eta(r)>0$ such that

$$
g(x) \geq \pi(A x)+\eta(r) d(x, \sigma(A x))^{2}, \quad \text { for all } x \in C \cap B(0, r),
$$

where $\pi(y)=\inf \{g(x): x \in C, A x=y\}$ and

$$
\sigma(y)=\operatorname{argmin}\{g(x): x \in C, A x=y\}, y \in A(C),
$$

(ii) the second-order set $S^{2}(\bar{x}, \xi)=\left\{z \in T^{2}(C ; \bar{x}, \xi):\langle\nabla g(\bar{x}), z\rangle+\left\langle\nabla Q_{\mu}(\bar{y}), A z\right\rangle=0\right\}$ is nonempty for each $\xi \in S(\bar{x})$.

Then the Gateaux directional derivative $\psi^{\prime}\left(Q_{\mu}, \bar{x} ; v\right)$ of $\psi$ at the pair $\left(Q_{\mu}, \bar{x}\right)$ exists for any direction $v \in T^{r}\left(K_{C} ; Q_{\mu}\right)$ and satisfies the formula in Theorem 4.1.

Moreover, condition (ii) is satisfied if $C$ is polyhedral, and (i)" is satisfied if $C$ is polyhedral and $g$ is linear or (convex) quadratic.

Proof. Let $v \in T^{r}\left(K_{C} ; Q_{\mu}\right)$. Since $Q_{\mu}$ is strongly convex on some open convex neighbourhood of $A \psi\left(Q_{\mu}\right)$, we infer from condition (i)" and Theorem 2.6 that condition (iii) of Theorem 4.1 is satisfied. Moreover, condition (i)" implies (i)' and, thus, Corollary 3.5 says that the second-order directional derivative $\varphi^{\prime \prime}\left(Q_{\mu} ; v\right)$ exists. Hence, the first part of the assertion follows from the proof of the previous theorem. If $C$ is polyhedral, we have $0 \in S^{2}(\bar{x}, \xi)$ for any $\xi \in S(\bar{x})$, and if, in addition, $g$ is convex quadratic, Proposition 2.2 implies condition (i $)^{\prime \prime}$ to hold.

We note that Example 3.7 shows that, in general, the directional differentiability property of $\psi$ gets lost at those pairs $\left(Q_{\mu}, \bar{x}\right), \bar{x} \in \psi\left(Q_{\mu}\right)$, where $Q_{\mu}$ is not strongly convex on some neighbourhood of $A \psi\left(Q_{\mu}\right)$. 
Finally, we turn to directional differentiability properties of $\psi$ where the derivatives exist uniformly with respect to directions taken from compact sets of certain functional spaces. For our first result we consider the space $C^{1}\left(\mathbb{R}^{s}\right)$ and equip the set $K_{C} \cap C^{1}$ with the $C^{1}$-topology.

Proposition 4.3 Let $Q_{\mu} \in K_{C} \cap C^{1}$ and assume that the general conditions on $g$, $Q_{\mu}$ and $C$ in Proposition 3.3 are satisfied. In addition, we suppose condition (ii) of Theorem 3.4 to be satisfied. Let $\bar{x} \in \psi\left(Q_{\mu}\right), t_{n} \rightarrow 0+$, and $\left(Q_{n}\right)$ be a sequence in $K_{C}$ such that $v_{n}:=\frac{1}{t_{n}}\left(Q_{n}-Q_{\mu}\right) \rightarrow v$ in $C^{1}\left(\mathbb{R}^{s}\right)$.

Then the upper set limit of the sequence $\left(\frac{1}{t_{n}}\left(\psi\left(Q_{\mu}+t_{n} v_{n}\right)-\bar{x}\right)\right.$ of closed convex subsets in $\mathbb{R}^{m}$, i.e., $\left.\limsup _{n \rightarrow \infty} \frac{1}{t_{n}}\left(\psi\left(Q_{\mu}+t_{n} v_{n}\right)-\bar{x}\right)\right)$, is contained in the closed convex set

$$
\operatorname{argmin}\left\{\frac{1}{2}\left\langle\nabla^{2} f(\bar{x}) \xi, \xi\right\rangle+\frac{1}{2}\left\langle\nabla^{2} Q_{\mu}(\bar{y}) A \xi, A \xi\right\rangle+\langle\nabla v(\bar{y}), A \xi\rangle: \xi \in S(\bar{x})\right\} .
$$

Proof. Let $D_{n}:=\frac{1}{t_{n}}\left(\psi\left(Q_{\mu}+t_{n} v_{n}\right)-\bar{x}\right)$ for all $n \in I N$ and let $\bar{\xi}$ belong to the upper set limit $\limsup _{n \rightarrow \infty} D_{n}$. Then there exist a subsequence (again denoted by $\left(D_{n}\right)$ ) and elements $\xi_{n} \in D_{n}$ such that $\xi_{n} \rightarrow \bar{\xi}$. Since $\bar{x}+t_{n} \xi_{n} \in \psi\left(Q_{\mu}+t_{n} v_{n}\right) \subseteq C$, we have that $\bar{\xi} \in T(C ; \bar{x})$. As in the proof of Theorem 3.4 we deduce that $\langle\nabla g(\bar{x}), \bar{\xi}\rangle+\left\langle\nabla Q_{\mu}(A \bar{x}), A \bar{\xi}\right\rangle=0$ and, thus, $\bar{\xi} \in S(\bar{x})$. By expanding $g$ and $Q_{\mu}$ as in the proof of Theorem 3.4, we also obtain analogously to $(3.1)$ :

$$
\begin{aligned}
\varphi\left(Q_{\mu}+t_{n} v_{n}\right)-\varphi\left(Q_{\mu}\right)-t_{n} \varphi^{\prime}\left(Q_{\mu} ; v_{n}\right) \\
=g\left(\bar{x}+t_{n} \xi_{n}\right)+Q_{\mu}\left(A\left(\bar{x}+t_{n} \xi_{n}\right)\right)-g(\bar{x})-Q_{\mu}(A \bar{x})-t_{n} v_{n}(A \bar{x}) \\
\geq \frac{1}{2} t_{n}^{2}\left\langle\nabla^{2} g(\bar{x}) \xi_{n}, \xi_{n}\right\rangle+\frac{1}{2} t_{n}^{2}\left\langle\nabla^{2} Q_{\mu}(A \bar{x}) A \xi_{n}, A \xi_{n}\right\rangle \\
+t_{n}\left(v_{n}\left(A\left(\bar{x}+t_{n} \xi_{n}\right)\right)-v_{n}(A \bar{x})\right)+o\left(\left\|t_{n} \xi_{n}\right\|^{2}\right) .
\end{aligned}
$$

After dividing by $t_{n}^{2}$ and taking the $\limsup _{n \rightarrow \infty}$ on both sides of the inequality, we obtain as in the proof of Theorem 3.8

$$
\begin{aligned}
& \limsup _{n \rightarrow \infty} \frac{1}{t_{n}^{2}}\left(\varphi\left(Q_{\mu}+t_{n} v_{n}\right)-\varphi\left(Q_{\mu}\right)-t_{n} \varphi^{\prime}\left(Q_{\mu} ; v_{n}\right)\right) \\
\geq & \frac{1}{2}\left\langle\nabla^{2} g(\bar{x}) \bar{\xi}, \bar{\xi}\right\rangle+\frac{1}{2}\left\langle\nabla^{2} Q_{\mu}(A \bar{x}) A \bar{\xi}, A \bar{\xi}\right\rangle+\langle\nabla v(A \bar{x}), A \bar{\xi}\rangle .
\end{aligned}
$$

Hence, we may conclude from (ii) and Proposition 3.3 that $\bar{\xi}$ belongs to the set $\operatorname{argmin}\left\{\frac{1}{2}\left\langle\nabla^{2} g(\bar{x}) \xi, \xi\right\rangle+\frac{1}{2}\left\langle\nabla^{2} Q_{\mu}(\bar{y}) A \xi, A \xi\right\rangle+\langle\nabla v(\bar{y}), A \xi\rangle: \xi \in S(\bar{x})\right\}$ and we are done.

Remark 4.4 The upper limit of the sequence $\left(\frac{1}{t_{n}}\left(\psi\left(Q_{\mu}+t_{n} v_{n}\right)-\bar{x}\right)\right.$ in Proposition 4.3 is nonempty if the mapping $d(\bar{x}, \psi(\cdot))$ from $K_{C}$ into the extended reals has the Lipschitzian property of Theorem 2.3 at $Q_{\mu}$. Indeed, we may select $x_{n} \in \psi\left(Q_{\mu}+t_{n} v_{n}\right)$ for large $n \in \mathbb{N}$, such that for some constants $\hat{L}>0$ and $r>0,\left\|\bar{x}-x_{n}\right\|=$ $d\left(\bar{x}, \psi\left(Q_{\mu}+t_{n} v_{n}\right)\right) \leq \hat{L} t_{n}\left\|v_{n}\right\|_{L, r}$. Hence, the sequence $\left(\frac{1}{t_{n}}\left(x_{n}-\bar{x}\right)\right)$ is bounded and 
has a convergent subsequence whose limit belongs to $\limsup _{n \rightarrow \infty} \frac{1}{t_{n}}\left(\psi\left(Q_{\mu}+t_{n} v_{n}\right)-\bar{x}\right)$. If the Lipschitz property of $d(\bar{x}, \psi(\cdot))$ is violated, the upper set limit may be empty. This is illustrated by Example 3.7, in which we have $\bar{x}=0, \psi\left(Q_{\mu}+t_{n} v\right)=\left\{\sqrt{t_{n}}\right\}$ and, thus, $\frac{1}{t_{n}}\left(\psi\left(Q_{\mu}+t_{n} v\right)-\bar{x}\right)=\left\{t_{n}^{-\frac{1}{2}}\right\}$.

In order to establish the semidifferentiability of $\psi$ at a pair $\left(Q_{\mu}, \bar{x}\right)$ belonging to the graph of $\psi$, it remains to show, according to Proposition 4.3, that the solution set

$$
\operatorname{argmin}\left\{\frac{1}{2}\left\langle\nabla^{2} g(\bar{x}) \xi, \xi\right\rangle+\frac{1}{2}\left\langle\nabla^{2} Q_{\mu}(\bar{y}) A \xi, A \xi\right\rangle+\langle\nabla v(\bar{y}), A \xi\rangle: \xi \in S(\bar{x})\right\}
$$

is contained in the lower set limit $\liminf _{n \rightarrow \infty} \frac{1}{t_{n}}\left(\psi\left(Q_{\mu}+t_{n} v_{n}\right)-\bar{x}\right)$, where $v_{n}:=\frac{1}{t_{n}}\left(Q_{n}-Q_{\mu}\right)$, $Q_{n} \in K_{C}$, for all $n \in I N$, and $\left(v_{n}\right)$ converges to $v$. To this end, a uniform quadratic growth condition of the objective functions $g(\cdot)+\left(Q_{\mu}+t_{n} v_{n}\right)(A \cdot)$, for large $n \in I N$, is significant. In view of Theorem 2.6, the uniform strong convexity of $Q_{\mu}$ and its approximations $Q_{n}$, for large $n \in \mathbb{N}$, is decisive for the growth condition. The next example and the following result show that the approximations $Q_{n}$ do not maintain the strong convexity property of $Q_{\mu}$ in general if the sequence $\left(Q_{n}\right)$ converges to $Q_{\mu}$ in $C^{1}\left(\mathbb{R}^{s}\right)$, but that the situation is much more advantageous when considering the $C^{1,1}$-topology.

Example 4.5 Let $Q_{\mu}(y)=y^{2}$, for all $y \in \mathbb{R}$, and $Q_{n}$ be the following differentiable convex functions

$$
Q_{n}(y):=\max \left\{0,-y-\frac{1}{n}\right\}^{2}+\max \left\{0, y-\frac{1}{n}\right\}^{2}, \quad \text { for all } y \in \mathbb{R}, n \in \mathbb{N} .
$$

Note that $Q_{n}(y)=0$, for all $y \in\left[-\frac{1}{n}, \frac{1}{n}\right]$, and $Q_{n}$ is not strongly convex for each $n \in I N$, but $\left(Q_{n}\right)$ converges to $Q_{\mu}$ in $C^{1}\left(\mathbb{R}^{s}\right)$.

Lemma 4.6 Let $Q_{\mu} \in K_{C} \cap C^{1,1}\left(\mathbb{R}^{s}\right)$ be strongly convex on some bounded convex set $U \subseteq \mathbb{R}^{s}$ (with some constant $\kappa>0$ ).

Then there exists a neighbourhood $\mathcal{N}$ of $Q_{\mu}$ in $C^{1,1}\left(\mathbb{R}^{s}\right)$ such that each function $Q$ belonging to $\mathcal{N}$ is strongly convex on $U$ with constant $\frac{\kappa}{2}$.

Proof. The strong convexity of $Q_{\mu}$ on $U$ (with constant $\kappa>0$ ) is equivalent to the condition $\left\langle\nabla Q_{\mu}(y)-\nabla Q_{\mu}(\tilde{y}), y-\tilde{y}\right\rangle \geq \kappa\|y-\tilde{y}\|^{2}$, for all $y, \tilde{y} \in U$. Let $r>0$ be chosen such that $\operatorname{cl} U \subseteq B(0, r)$ and let $\mathcal{N}$ be a neighbourhood of $Q_{\mu}$ in $C^{1,1}\left(\mathbb{R}^{s}\right)$ having the property $\left\|\nabla\left(Q_{\mu}-Q\right)\right\|_{L, r} \leq \frac{\kappa}{2}$, for all $Q \in \mathcal{N}$. Let $y, \tilde{y} \in U$ with $y \neq \tilde{y}$. Then we obtain for any $Q \in \mathcal{N}$,

$$
\begin{aligned}
\kappa & \leq \frac{\left\langle\nabla Q_{\mu}(y)-\nabla Q_{\mu}(\tilde{y}), y-\tilde{y}\right\rangle}{\|y-\tilde{y}\|^{2}} \\
& =\frac{\langle\nabla Q(y)-\nabla Q(\tilde{y}), y-\tilde{y}\rangle}{\|y-\tilde{y}\|^{2}}+\frac{\left\langle\nabla\left(Q_{\mu}-Q\right)(y)-\nabla\left(Q_{\mu}-Q\right)(\tilde{y}), y-\tilde{y}\right\rangle}{\|y-\tilde{y}\|^{2}} \\
& \leq \frac{\langle\nabla Q(y)-\nabla Q(\tilde{y}), y-\tilde{y}\rangle}{\|y-\tilde{y}\|^{2}}+\frac{\left\|\nabla\left(Q_{\mu}-Q\right)(y)-\nabla\left(Q_{\mu}-Q\right)(\tilde{y})\right\|}{\|y-\tilde{y}\|^{2}} \\
& \leq \frac{\langle\nabla Q(y)-\nabla Q(\tilde{y}), y-\tilde{y}\rangle}{\|y-\tilde{y}\|^{2}}+\left\|\nabla\left(Q_{\mu}-Q\right)\right\|_{L, r}
\end{aligned}
$$


and, hence

$$
\frac{\kappa}{2}\|y-\tilde{y}\|^{2} \leq\langle\nabla Q(y)-\nabla Q(\tilde{y}), y-\tilde{y}\rangle
$$

This means that $Q$ is strongly convex on $U$ with constant $\frac{\kappa}{2}$.

Now we are able to show that the solution set mapping $\psi$ is semidifferentiable on $K_{C} \cap C^{1,1}$ at some pairs $\left(Q_{\mu}, \bar{x}\right), \bar{x} \in \psi\left(Q_{\mu}\right)$, into any direction $v$ from the tangent cone $T\left(K_{C} \cap C^{1,1} ; Q_{\mu}\right)$ to $K_{C} \cap C^{1,1}\left(\mathbb{R}^{s}\right)$ at $Q_{\mu}$ in $C^{1,1}\left(\mathbb{R}^{s}\right)$. The assumptions are essentially the same as in Theorem 4.2.

Theorem 4.7 Let $Q_{\mu} \in K_{C} \cap C^{1,1}$ and assume that $\psi\left(Q_{\mu}\right)$ is nonempty, bounded. Let $g$ be twice continuously differentiable, $Q_{\mu}$ be strongly convex on some open convex neighbourhood $U$ of $A \psi\left(Q_{\mu}\right)$ and twice continuously differentiable at $\bar{y}$, where $\{\bar{y}\}=$ $A \psi\left(Q_{\mu}\right)$. Assume that, for each $r>0$, there exist constants $L>0$ and $\eta(r)>0$ such that the following condition (i)" is satisfied for $\pi(y)=\inf \{g(x): x \in C, A x=y\}$ and $\sigma(y)=\operatorname{argmin}\{g(x): x \in C, A x=y\} \quad(y \in A(C)):$

$(\mathrm{i})^{\prime \prime} \quad d_{H}(\sigma(y), \sigma(\tilde{y})) \leq L\|y-\tilde{y}\|, \quad$ for all $y, \tilde{y} \in A(C)$,

$$
g(x) \geq \pi(A x)+\eta(r) d(x, \sigma(A x))^{2}, \quad \text { for all } x \in C \cap B(0, r) .
$$

Then the solution set mapping $\psi$ from $K_{C} \cap C^{1,1}$ into $\mathbb{R}^{m}$ is semidifferentiable at any pair $\left(Q_{\mu}, \bar{x}\right), \bar{x} \in \psi\left(Q_{\mu}\right)$, such that $S^{2}(\bar{x}, \xi)$ is nonempty for each $\xi \in S(\bar{x})$, and into any direction $v \in T\left(K_{C} \cap C^{1,1} ; Q_{\mu}\right)$, i.e., for any such $\bar{x}$ and $v, t_{n} \rightarrow 0+$, and $\left(Q_{n}\right)$ in $K_{C} \cap C^{1,1}$ with $v_{n}=\frac{1}{t_{n}}\left(Q_{n}-Q_{\mu}\right) \rightarrow v$ in $C^{1,1}\left(\mathbb{R}^{s}\right)$ the set limit

$D \psi\left(Q_{\mu}, \bar{x} ; v\right)=\lim _{n \rightarrow \infty} \frac{1}{t_{n}}\left(\psi\left(Q_{\mu}+t_{n} v_{n}\right)-\bar{x}\right)$

exists. The semiderivative $D \psi\left(Q_{\mu}, \bar{x} ; v\right)$ is equal to the set

$\operatorname{argmin}\left\{\frac{1}{2}\left\langle\nabla^{2} g(\bar{x}) \xi, \xi\right\rangle+\frac{1}{2}\left\langle\nabla^{2} Q_{\mu}(\bar{y}) A \xi, A \xi\right\rangle+\langle\nabla v(\bar{y}), A \xi\rangle: \xi \in S(\bar{x})\right\}$.

Moreover, $\psi$ is semidifferentiable at any pair $\left(Q_{\mu}, \bar{x}\right), \bar{x} \in \psi\left(Q_{\mu}\right)$, into any direction $v \in T\left(K_{C} \cap C^{1,1} ; Q_{\mu}\right)$ if $C$ is polyhedral. Condition (i)" is satisfied if $C$ is polyhedral and $g$ is linear or (convex) quadratic.

Proof. Let $\bar{x} \in \psi\left(Q_{\mu}\right)$ be such that $S^{2}(\bar{x}, \xi)$ is nonempty for each $\xi \in S(\bar{x})$, $v \in T\left(K_{C} \cap C^{1,1} ; Q_{\mu}\right)$, and $v_{n}=\frac{1}{t_{n}}\left(Q_{n}-Q_{\mu}\right) \rightarrow v$ in $C^{1,1}\left(\mathbb{R}^{s}\right)$, where $t_{n} \rightarrow 0+$ and $\left(Q_{n}\right)$ is a sequence in $K_{C} \cap C^{1,1}$. We may assume that $U$ is bounded. Since $\left(Q_{n}\right)$ converges to $Q_{\mu}$ in $C^{1,1}\left(\mathbb{R}^{s}\right)$, we obtain from Lemma 4.6 that there exists an $n_{0} \in I N$, such that $Q_{n}$ is strongly convex on $U$ for each $n \geq n_{0}$ with a uniform constant $\kappa>0$. Moreover, we choose $n_{0}$ sufficiently large such that $\psi\left(Q_{n}\right)$ is nonempty, for each $n \geq n_{0}$. Arguing as in the proof of Theorem 2.6, we obtain a constant $c>0$ and a neighbourhood $V$ of $\psi\left(Q_{n}\right)$ such that the growth condition

$$
g(x)+Q_{n}(A x) \geq \varphi\left(Q_{n}\right)+c d\left(x, \psi\left(Q_{n}\right)\right)^{2}
$$


holds for all $x \in C \cap V$ and $n \geq n_{0}$.

Let $\bar{\xi} \in S(\bar{x})$ be a minimizer of the function $\frac{1}{2}\left\langle\nabla^{2} g(\bar{x}) \xi, \xi\right\rangle+\frac{1}{2}\left\langle\nabla^{2} Q_{\mu}(\bar{y}) A \xi, A \xi\right\rangle+$ $\langle\nabla v(\bar{y}), A \xi\rangle$ subject to $\xi \in S(\bar{x})$. Because of Proposition 4.3 it remains to show that $\bar{\xi}$ belongs to the lower limit $\liminf _{n \rightarrow \infty} \frac{1}{n}\left(\psi\left(Q_{\mu}+t_{n} v_{n}\right)-\bar{x}\right)=\liminf _{n \rightarrow \infty} \frac{1}{t_{n}}\left(\psi\left(Q_{n}\right)-\bar{x}\right)$. Since $\bar{\xi} \in S(\bar{x})$, there exists an element $z \in S^{2}(\bar{x}, \bar{\xi})$ and a sequence $\left(z_{n}\right)$ converging to $z$ with $\bar{x}+t_{n} \bar{\xi}+t_{n}^{2} z_{n} \in C$ for all $n \in \mathbb{I N}$. As in the proof of Theorem 4.1 it suffices to show that

$$
\lim _{n \rightarrow \infty} \frac{1}{t_{n}} d\left(\bar{x}+t_{n} \bar{\xi}+t_{n}^{2} z_{n}, \psi\left(Q_{n}\right)\right)=0 .
$$

By using the above growth condition and by expanding the function $g$ and $Q_{\mu}$, we obtain as in the proof of Theorem 4.1

$$
\begin{aligned}
& c d\left(\bar{x}+t_{n} \bar{\xi}+t_{n}^{2} z_{n}, \psi\left(Q_{n}\right)\right) \leq g\left(\bar{x}+t_{n} \bar{\xi}+t_{n}^{2} z_{n}\right)+Q_{n}\left(A\left(\bar{x}+t_{n} \bar{\xi}+t_{n}^{2} z_{n}\right)\right)-\varphi\left(Q_{n}\right) \\
= & g\left(\bar{x}+t_{n} \bar{\xi}+t_{n}^{2} z_{n}\right)+Q_{\mu}\left(A\left(\bar{x}+t_{n} \bar{\xi}+t_{n}^{2} z_{n}\right)\right)-g(\bar{x})-Q_{\mu}(A \bar{x}) \\
& -\left(\varphi\left(Q_{n}\right)-\varphi\left(Q_{\mu}\right)-t_{n} \varphi^{\prime}\left(Q_{\mu} ; v_{n}\right)\right)+t_{n}\left(v_{n}\left(A\left(\bar{x}+t_{n} \bar{\xi}+t_{n}^{2} z_{n}\right)\right)-v_{n}(A \bar{x})\right)
\end{aligned}
$$

and

$$
\begin{aligned}
& \limsup _{n \rightarrow \infty} \frac{c}{t_{n}^{2}} d\left(\bar{x}+t_{n} \bar{\xi}+t_{n}^{2} z_{n}, \psi\left(Q_{n}\right)\right)^{2} \\
\leq & \langle\nabla g(\bar{x}), z\rangle+\left\langle\nabla Q_{\mu}(\bar{y}), A z\right\rangle+\frac{1}{2}\left\langle\nabla^{2} g(\bar{x}) \bar{\xi}, \bar{\xi}\right\rangle \\
& +\frac{1}{2}\left\langle\nabla^{2} Q_{\mu}(\bar{y}) A \bar{\xi}, A \bar{\xi}\right\rangle-\varphi^{\prime \prime}\left(Q_{\mu} ; v\right)+\langle\nabla v(\bar{y}), A \bar{\xi}\rangle=0 .
\end{aligned}
$$

This implies $\bar{\xi} \in \liminf _{n \rightarrow \infty} \frac{1}{t_{n}}\left(\psi\left(Q_{n}\right)-\bar{x}\right)$ and the semidifferentiability of $\psi$ at $\left(Q_{\mu}, \bar{x}\right)$ in direction $v$ is shown. The remaining part of the assertion follows as in the proof of Theorem 4.2.

For the linear-quadratic case, the essential assumptions in Theorem 4.7 are the strong convexity of $Q_{\mu}$, and the smoothness properties of $Q_{\mu}$ and its perturbations $Q$, respectively. While criteria for strong convexity were already discussed in Section 2, we now close this section by adding some comments on $C^{1,1}$ - and $C^{2}$-properties of expected recourse functions.

Remark 4.8 Assume (A1) - (A3) and $\mu$ to have a density with respect to the Lebesgue measure on $\mathbb{R}^{s}$. Then the function $Q_{\mu}$ in (1.2) is continuously differentiable on $\mathbb{R}^{s}$ and its gradient is of the form $\nabla Q_{\mu}(y)=\sum_{i=1}^{\ell} d_{i} \mu\left(y+B_{i}\left(\mathbb{R}_{+}^{s}\right)\right)$, for all $y \in \mathbb{R}^{s}$, where $B_{i}$, $i=1, \ldots, \ell$, are certain basis submatrices of the recourse matrix $W$ such that the simplicial cones $B_{i}\left(\mathbb{R}_{+}^{s}\right), i=1, \ldots, \ell$, are linearity regions of $\tilde{Q}$ and $d_{i}$ is the gradient of $\tilde{Q}$ on int $B_{i}\left(\mathbb{R}_{+}^{s}\right), i=1, \ldots, \ell$ (cf. [14], [36]). Denoting by $F_{\mu}$ the distribution function of $\mu$ and using the formula

$$
\mu\left(y+B\left(\mathbb{R}_{+}^{s}\right)\right)=F_{\mu \circ(-B)}\left(-B^{-1} y\right), \quad \text { for all } y \in \mathbb{R}^{s},
$$

for any nonsingular $(s, s)$-matrix $B, C^{1,1}$ - and $C^{2}$-properties of $Q_{\mu}$ may thus be formulated in terms of Lipschitz and differentiability properties of the distribution functions 
$F_{\mu \circ\left(-B_{i}\right)}$ to the linear transforms $\mu \circ\left(-B_{i}\right), i=1, \ldots, \ell$, of the measure $\mu$.

The distribution function $F_{\mu}$ of a probability measure $\mu$ on $\mathbb{R}^{s}$ is locally Lipschitzian if all one-dimensional marginal distribution functions of $\mu$ are locally Lipschitzian (cf. [24], [35]). $F_{\mu}$ is continuously differentiable if $\mu$ has a continuous density function and all one-dimensional marginal distribution functions of $\mu$ are continuously differentiable (cf. [19], [35]). If $\mu$ has a continuous density function, then $\mu \circ B$ has a continuous density for any nonsingular $(s, s)$-matrix $B$, too. Hence, we may conclude, for instance, that $Q_{\mu}$ belongs to $C^{1,1}\left(\mathbb{R}^{s}\right)$ (and $C^{2}\left(\mathbb{R}^{s}\right)$ ) if $\mu$ has a (continuous) density and the above-mentioned conditions on the one-dimensional marginal distribution functions for $F_{\mu \circ B}$ belonging to $C^{0,1}\left(\mathbb{R}^{s}\right)$ (and $C^{1}\left(\mathbb{R}^{s}\right)$, respectively) are satisfied for any nonsingular $(s, s)$-matrix $B$. This criterion is particularly useful for probability distributions $\mu$ which have the property that all one-dimensional marginal distributions of $\mu$ and all linear transforms $\mu \circ B$, for all nonsingular matrices $B$, belong to the same class of measures. For instance, all multivariate normal and all logarithmic concave probability measures (e.g. [14]) form classes having this property.

Acknowledgement: The authors wish to thank Alexander Shapiro (Georgia Institute of Technology, Atlanta) and René Henrion (WIAS Berlin) for beneficial discussions.

\section{References}

[1] Z. Artstein and R.J.-B. Wets, Stability results for stochastic programs and sensors, allowing for discontinuous objective functions, SIAM Journal on Optimization 4 (1994), 537-550.

[2] J.-P. Aubin and H. Frankowska, Set-Valued Analysis, Birkhäuser, Boston 1990.

[3] A. Auslender and R. Cominetti, First and second order sensitivity analysis of nonlinear programs under directional constraint qualification conditions, Optimization 21 (1990), 351-363.

[4] A. Auslender and R. Cominetti, A comparative study of multifunction differentiability with applications in mathematical programming, Mathematics of Operations Research 16 (1991), 240-258.

[5] A. Ben-Tal and J. Zowe, Directional derivatives in nonsmooth optimization, Journal of Optimization Theory and Applications 47 (1985), 483-490.

[6] J.F. Bonnans and R. Cominetti, Perturbed optimization in Banach spaces I: A general theory based on a weak directional constraint qualification, SIAM Journal on Control and Optimization 34 (1996), 1151-1171.

[7] J.F. Bonnans and A.D. Ioffe, Quadratic growth and stability in convex programming problems with multiple solutions, Journal of Convex Analysis 2 (1995), 41-57. 
[8] J.F. Bonnans and A. Shapiro, Optimization problems with perturbations, a guided tour, Rapport de Recherche INRIA 2872, Avril 1996.

[9] F.H. Clarke, Optimization and Nonsmooth Analysis, Wiley, New York 1983.

[10] R. Cominetti, Metric regularity, tangent sets and second-order optimality conditions, Applied Mathematics and Optimization 21 (1990), 265-287.

[11] D. Dentcheva, Differentiable selections of set-valued mappings with application in stochastic programming, Humboldt-Universität Berlin, Institut für Mathematik, Preprint Nr. 96-28, 1996.

[12] D. Dentcheva, W. Römisch and R. Schultz, Strong convexity and directional differentiability of marginal values in two-stage stochastic programming, in: Stochastic Programming (K. Marti and P. Kall, Eds.), Lecture Notes in Economics and Mathematical Systems Vol. 423, Springer-Verlag, Berlin 1995, 8-21.

[13] J. Dupačová, Stability and sensitivity analysis for stochastic programming, Annals of Operations Research 27 (1990), 115-142.

[14] P. Kall, Stochastic Linear Programming, Springer-Verlag, Berlin 1976.

[15] A.J. King, Generalized delta theorems for multivalued mappings and measurable selections, Mathematics of Operations Research 14 (1989), 720-736.

[16] A.J. King and R.T. Rockafellar, Sensitivity analysis for nonsmooth generalized equations, Mathematical Programming 55 (1992), 193-212.

[17] A.J. King and R.T. Rockafellar, Asymptotic theory for solutions in statistical estimation and stochastic programming, Mathematics of Operations Research 18 (1993), 148-162.

[18] D. Klatte and G. Thiere, Error bounds for solutions of linear equations and inequalities, ZOR-Mathematical Methods of Operations Research 41 (1995), 191214 .

[19] K. Marti, Approximationen der Entscheidungsprobleme mit linearer Ergebnisfunktion und positiv homogener, subadditiver Verlustfunktion, Zeitschrift für Wahrscheinlichkeitstheorie und verwandte Gebiete 31 (1975), 203-233.

[20] B.S. Mordukhovich, Stability theory for parametric generalized equations and variational inequalities via nonsmooth analysis, Transactions of the American Mathematical Society 343 (1994), 609-657.

[21] J.-P. Penot, Differentiability of relations and differential stability of perturbed optimization problems, SIAM Journal on Control and Optimization 22 (1984), $529-551$.

[22] S.M. Robinson, Strongly regular generalized equations, Mathematics of Operations Research 5 (1980), 43-62. 
[23] R.T. Rockafellar, Proto-differentiability of set-valued mappings and its applications in optimization, Annales de l'Institut Henri Poincare, Analyse Non Linéaire (H. Attouch et al. Eds.), Gauthier-Villares, Paris, Supplément au 6 (1989), 449482 .

[24] W. Römisch and R. Schultz, Stability of solutions for stochastic programs with complete recourse, Mathematics of Operations Research 18 (1993), 590-609.

[25] W. Römisch and R. Schultz, Lipschitz stability for stochastic programs with complete recourse, SIAM Journal on Optimization 6 (1996), 531-547.

[26] R.Y. Rubinstein and A. Shapiro, Discrete Event Systems. Sensitivity Analysis and Stochastic Optimization by the Score Function Method, Wiley, Chichester 1993.

[27] R. Schultz, Strong convexity in stochastic programs with complete recourse, Journal of Computational and Applied Mathematics 56 (1994), 3-22.

[28] A. Seeger, Second order directional derivatives in parametric optimization problems, Mathematics of Operations Research 13 (1988), 628-645.

[29] A. Shapiro, Sensitivity analysis of nonlinear programs and differentiability properties of metric projections, SIAM Journal on Control and Optimization 26 (1988), 628-645.

[30] A. Shapiro, On concepts of directional differentiability, Journal of Optimization Theory and Applications 66 (1990), 477-487.

[31] A. Shapiro, On differential stability in stochastic programming, Mathematical Programming 47 (1990), 107-116.

[32] A. Shapiro, Asymptotic analysis of stochastic programs, Annals of Operations Research 30 (1991), 169-186.

[33] A.W. van der Vaart and J.A. Wellner, Weak Convergence and Empirical Processes, Springer Series in Statistics, Springer-Verlag, New York 1996.

[34] D. Walkup and R.J.-B. Wets, A Lipschitzian characterization of convex polyhedra, Proceedings of the American Mathematical Society 23 (1969), 167-173.

[35] J. Wang, Distribution sensitivity analysis for stochastic programs with complete recourse, Mathematical Programming 31 (1985), 286-297.

[36] R.J.-B. Wets, Stochastic programs with fixed recourse: the equivalent deterministic program, SIAM Review 16 (1974), 309-339.

[37] R.J.-B. Wets, Stochastic programming, in: Handbooks in Operations Research and Management Science. Vol. 1, Optimization (G.L. Nemhauser, A.H.G. Rinnoy Kan, M.J. Todd, Eds.), North-Holland, Amsterdam 1989, 573-629. 